\title{
Role of rare events in the pinning problem
}

\author{
M. Buchacek (1), V. B. Geshkenbein (ㄷ, and G. Blatter (1) \\ Institute for Theoretical Physics, ETH Zurich, 8093 Zurich, Switzerland
}

(Received 18 July 2020; accepted 23 October 2020; published 20 November 2020)

\begin{abstract}
Type II superconductors exhibit a fascinating phenomenology that is determined by the dynamical properties of the vortex matter hosted by the material. A crucial element in this phenomenology is vortex pinning by material defects, e.g., immobilizing vortices at small drives and thereby guaranteeing dissipation-free current flow. Pinning models for vortices and other topological defects, such as domain walls in magnets or dislocations in crystals, come in two standard variants: (1) weak-collective pinning, where individual weak defects are unable to pin, while the random accumulation of many force centers within a collective pinning volume combines into an effective pin, and (2) strong pinning, where strong defects produce large vortex displacements and bistabilities that lead to pinning on the level of individual defects. The transition between strong and weak pinning is quantified by the Labusch criterion $\kappa \approx f_{p} / \bar{C} \xi=1$, where $f_{p}$ and $\bar{C}$ are the force of one defect and the effective elasticity of the vortex lattice, respectively ( $\xi$ is the coherence length). Here, we show that a third generic type of pinning becomes dominant when the pinning force $f_{p}$ enters the weak regime, the pinning by rare events. We find that within an intermediate regime $1 / 2<\kappa<1$, compact pairs of weak defects define strong pinning clusters that extend the mechanism of strong pinning into the weak regime. We present a detailed analysis of this cluster-pinning mechanism and show that its pinning force density parametrically dominates over the weak pinning result. The present work is a first attempt to include correlations between defects into the discussion of strong pinning.
\end{abstract}

DOI: 10.1103/PhysRevResearch.2.043266

\section{INTRODUCTION}

Broken-symmetry phases, as they appear in superconducting, magnetic, or density wave systems, exhibit physical properties on top of those originating from the underlying material. Typically, these ordered phases develop topological excitations (or defects) that govern the material properties, e.g., vortices in superconductors [1] or domain walls in magnets [2,3]. Remarkably, it is the interaction between the material's and the topological defects that determines the static and dynamical properties of the latter, with pinning immobilizing vortices in superconductors guaranteeing the material's dissipation-free current transport [4,5] and fixing domain walls in the magnet determining its coercive field [6]. On the fundamental side, pinning of topological defects constitutes a rich branch of disordered statistical physics with challenging phase-space and ergodicity properties, including the phenomenon of glassiness [7,8].

Traditionally, pinning in such systems was thought of as due to large ensembles of weak defects; the ensuing collective pinning theory [5,9-11] has become a common framework for the description of pinning of superconducting vortices [7,12-14], magnetic domain walls [15-17], charge density

Published by the American Physical Society under the terms of the Creative Commons Attribution 4.0 International license. Further distribution of this work must maintain attribution to the author $(s)$ and the published article's title, journal citation, and DOI. waves (CDWs) [18,19] and other types of elastic media [20]. At the same time, an alternative viewpoint describing pinning due to a low density of strong centers was proposed early on; see Refs. [4,5]. Recently, this strong pinning scenario has attracted increasing attention, particularly in studies of charge density waves [19,21,22] and of magnetic flux-line lattices [23-27]. Although some effort has been made to qualitatively understand the crossover between the two regimes [28,29], a quantitative model describing this transition has not been developed so far. In this paper, we describe a regime with a different pinning mechanism that appears at the crossover between the two theories. We show that in a considerable part of the weak region, pinning is dominated by defect clusters cooperating on short distances and forming strong pinning centers that are described with the tools of strong pinning theory. The dominance of these strong pinning small pairs over the weak-collective ensembles can be traced back to the dispersive nature of the vortex elasticity. Pinning by rare events then interpolates between the strong pinning of individual defects and the random sum of weak pinning forces due to the many defects within the Larkin domains of collective pinning theory, as illustrated in Fig. 1.

The central problem arising in studies of pinned systems is the determination of the maximal driving (or critical) force density $F_{c}$ below which the system remains immobilized. This critical force is determined by the competition between the pinning centers characterized by their density $n_{p}$ and individual forces $f_{p}$ and the elastic properties of the manifold. In the present study, we focus on the vortex lattice [1] formed by flux lines or vortices, each carrying a superconducting 


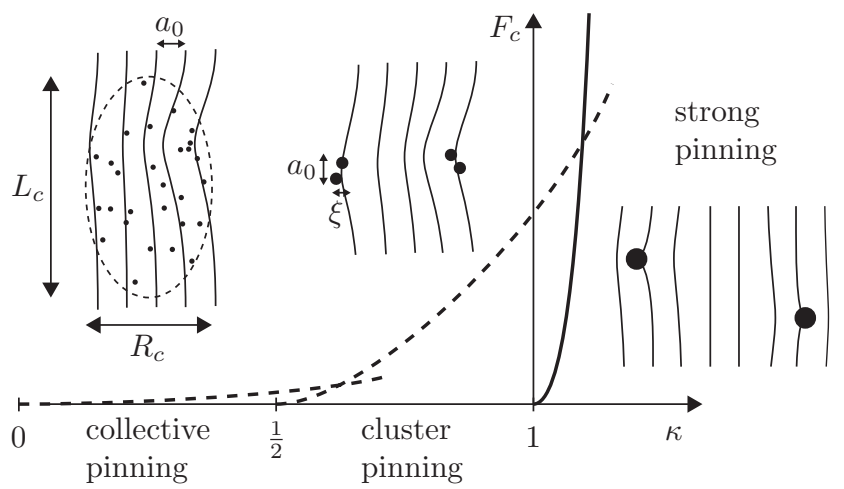

FIG. 1. Pinning mechanisms for flux lattices in type II superconductors in the regime of low defect density $n_{p} a_{0} \xi^{2} \ll 1$. Shown is the critical force density $F_{c}$ as a function of pinning strength $\kappa \propto f_{p} / \bar{C} \xi$. For small $\kappa \rightarrow 0$, pinning arises due to the collective action of a large number of defects within the Larkin volume $V_{c} \sim R_{c}^{2} L_{c}$, resulting in the collective pinning force density $F_{\text {coll }} \sim(\xi / \lambda)^{2} \kappa^{3} f_{p} n_{p}\left(n_{p} a_{0} \xi^{2}\right)$. For intermediate values $1 / 2<\kappa<1$, pairs of defects in close proximity form strong pinning clusters that produce the cluster pinning force density $F_{\text {clust }} \sim\left(\xi / a_{0}\right)^{2}\left(\kappa-\frac{1}{2}\right)^{4} f_{p} n_{p}\left(n_{p} a_{0} \xi^{2}\right)$; the latter dominates over the collective pinning result for a Labusch parameter $\kappa>$ $1 / 2+O\left[\left(a_{0} / \lambda\right)^{1 / 2}\right]$. For $\kappa \gtrsim 1$, pinning is strong, with the pinning force density due to individual defects rising as $\left(\xi / a_{0}\right)^{2}(\kappa-1)^{2} f_{p} n_{p}$; the latter dominates over the cluster pinning when increasing $\kappa$ beyond unity by the amount $\left(n_{p} a_{0} \xi^{2}\right)^{1 / 2}$.

flux quantum $\Phi_{0}$ and characterized by a line energy $\varepsilon_{0}=$ $\left(\Phi_{0} / 4 \pi \lambda\right)^{2}$ ( $\lambda$ denotes the London penetration depth). The effective elasticity $\bar{C} \sim \varepsilon_{0} / a_{0}$, with $a_{0}$ the distance between vortices, captures the full elastic properties of the vortex lattice that combines the line tension and the interaction between vortices. The competition between pinning and elastic forces then can be quantified by the dimensionless Labusch parameter [4] $\kappa \sim f_{p} / \xi \bar{C}$, where $\xi$ denotes the coherence length (or vortex diameter) in the superconductor. When $\kappa$ increases beyond unity, individual pins change from weak to strong. The three scenarios, strong, weak-collective, and cluster pinning as illustrated in Fig. 1 then provide different mechanisms and scaling laws for the critical force density $F_{c}$.

The strong pinning paradigm rests on the assumption of a low defect density $n_{p}$, such that $\kappa n_{p} a_{0} \xi^{2}<1$ [28], and strong defects, i.e., $\kappa>1$. In this setting, material defects act independently, resulting in a critical force density $F_{c} \propto n_{p}$ that is linear in the density $n_{p}$ of pinning centers. The task of calculating $F_{c}$ then simplifies considerably and even allows for a quantitative treatment: as defects act independently, the calculation of their contribution to the critical force density $F_{c}$ boils down to an effective single-particle problem where a strong defect interacts with an elastic manifold. The competition between potential and elastic forces does, however, add quite some complexity to the problem, with strong pinning inducing plastic deformations and bistable (pinned and free) states of the elastic manifold [5,19,21,30]. The nonsymmetric occupation of these bistable solutions then generates a finite pinning force, with the critical force density derived from the maximally asymmetric occupation of metastable states given by $F_{c} \sim\left(S_{\text {trap }} / a_{0}^{2}\right) n_{p} f_{p} \sim\left(\kappa \xi^{2} / a_{0}^{2}\right) n_{p} f_{p}$; here $S_{\text {trap }} / a_{0}^{2}$ defines the fraction of vortices falling into the defect trapping area $S_{\text {trap }} \sim \kappa \xi^{2}$ with longitudinal and transverse dimensions $\sim \kappa \xi$ and $\sim \xi[30,31]$.

Weak-collective pinning instead, relies on the joint action of many defects, as individual weak pins with $\kappa<$ 1 cannot hold the manifold. In the weak-collective pinning scenario, distant defects act with random forces on the manifold and their (random) addition within the Larkin volume $V_{c} \sim \lambda^{3}\left(\lambda / a_{0}\right) /\left(\kappa^{2} n_{p} a_{0} \xi^{2}\right)^{3}$ (that contains a large number of pins) produces a critical force density $F_{c} \sim$ $\left[\left(\xi^{2} / a_{0}^{2}\right) n_{p} f_{p}^{2} V_{c}\right]^{1 / 2} / V_{c} \sim\left(\xi^{2} / \lambda^{2}\right) \kappa^{3}\left(n_{p} a_{0} \xi^{2}\right) n_{p} f_{p}$, where the factor $\xi^{2} / a_{0}^{2}$ accounts for the fraction of defects within $V_{c}$ that overlap with the vortex cores. In fact, the collective force randomly accumulated in the Larkin volume $V_{c}$ produces an effectively strong pin [28] that satisfies the Labusch criterion $\kappa\left(V_{c}\right)=1$.

In the present paper, we study the crossover between the strong and weak-collective pinning mechanisms near $\kappa \sim 1$; this study leads us to the mechanism of pinning by rare events. Pairs of defects that reinforce one another appear with relative probability $n_{p}^{2}$ and thus potentially compete with the force generated in the weak pinning scenario. In identifying suitable pairs, we find that nearby defects within the action volume $\xi^{2} a_{0}$ of one defect define the relevant clusters; the density of such clusters then is given by $\left(n_{p} a_{0} \xi^{2}\right) n_{p}$. Defects in one cluster act cooperatively rather then competitively. For defects with a pinning strength $1 / 2<\kappa<1$, such neighboring pairs jointly produce a strong defect with $2 \kappa>1$. Applying the strong pinning formalism to these strong cluster defects then produces a critical force density $F_{c} \sim\left(\xi^{2} / a_{0}^{2}\right)\left(n_{p} a_{0} \xi^{2}\right) n_{p} f_{p}$ that is larger than the weak-collective force density by a factor $\left(\lambda / a_{0}\right)^{2}$. This factor is a consequence of the dispersive nature of the tilt elasticity $c_{44}(\mathbf{k})$ : while (nondispersive) collective pinning involves the large Larkin scale $R_{c}>\lambda$, cluster pinning appears on short distances below $a_{0}$ and hence involves the line rather than the bulk elasticity. Hence, we find a transition region in the pinning strength $\kappa$ where rare events, neighboring defects forming a strong pinning cluster, determine the critical force density $F_{c}$.

The relevance of rare events has been pointed out before in the context of charge density wave pinning [32], where an analysis in $D>4$ dimensions demonstrated the irrelevance of weak-collective pinning. Instead, a finite but exponentially small (in the disorder strength) pinning force density was found that originates from rare regions with anomalously coherent pinning. In our case, we deal with a $D=3$-dimensional vortex lattice, where both types of pinning, weak-collective and rare events, contribute simultaneously, with the rare events identified as small defect pairs.

The paper is organized as follows: in Sec. II, we discuss the formalism used in the description of vortex pinning for the generic case of an isotropic material and briefly present the main steps in the derivation of the pinning force density $F_{c}$ in the strong and weak pinning scenarios and for the newly introduced framework of pinning by close pairs of defects. In our analysis of weak and pair/cluster pinning, we assume an uncorrelated distribution of defects; alternative scenarios are possible where defects are spatially correlated, e.g., as a result of material growth and or special treatment. Well-known 
examples are grain boundaries in cuprate superconductors or columnar defects due to heavy-ion irradiation; see Ref. [7] for a detailed discussion. In Sec. III, we first introduce the general two-defect problem for pairs of any size. In the overview section III C, we identify the strong pinning pairs and discuss their contribution to the pinning force density as a function of the spatial separation between the defects constituting the pair. We show that pairs of distant defects provide a smaller contribution, justifying the assumption of dominant pinning by rare clusters of close defect pairs. We proceed with a detailed analytical derivation of our results, involving an indepth discussion of the effective anisotropic pinning potential of defect pairs (Sec. III D), the effective Labusch parameter of defect pairs in Sec. III E, and the average pinning force of defect pairs in Sec. III F including a comparison to numerical results. Finally, in Sec. IV, we summarize our results and place them into context, including also some further directions of research.

\section{VORTEX LATTICE PINNING}

The pinning of a vortex lattice is an example of the $(D+n)$-dimensional random manifold problem; the latter describes a $D$-dimensional elastic manifold with elasticity $c$ parametrized by $\rho \in \mathbb{R}^{D}$ that is distorted with an $n$ dimensional displacement field $\mathbf{u}(\rho) \in \mathbb{R}^{n}$ due to a pinning potential $\varepsilon_{\text {pin }}(\boldsymbol{\rho}, \mathbf{u})$. Assuming small distortions, the generic Hamiltonian

$$
H=\int d^{D} \rho\left[\frac{c}{2} \sum_{i, \alpha}\left(\partial_{\rho_{i}} u_{\alpha}\right)^{2}+\varepsilon_{\text {pin }}(\boldsymbol{\rho}, \mathbf{u})\right]
$$

describes this type of systems. Minimizing Eq. (1) yields the equation for the displacement field in the form

$$
\mathbf{u}(\boldsymbol{\rho})=\int d^{D} \boldsymbol{\rho}^{\prime} G\left(\boldsymbol{\rho}-\boldsymbol{\rho}^{\prime}\right)\left\{-\nabla_{\mathbf{u}} \varepsilon_{\mathrm{pin}}\left[\boldsymbol{\rho}^{\prime}, \mathbf{u}\left(\boldsymbol{\rho}^{\prime}\right)\right]\right\},
$$

with the Green's function $G(\boldsymbol{\rho})$; in reciprocal space, $G(\mathbf{k})=$ $1 / c \mathbf{k}^{2}$. Going over to the vortex lattice, the single elastic constant $c$ of the generic manifold is replaced by an elastic matrix that includes anisotropic and dispersive tilt $\left(c_{44}\right)$, compression $\left(c_{11}\right)$, and shear $\left(c_{66}\right)$ moduli [7]. In the following, we first discuss the relevant properties of the real-space Green's function $G(\rho)$ for our vortex problem and then turn to the peculiarities of the disorder potential $\varepsilon_{\text {pin }}(\rho, \mathbf{u})$ for the weak and strong pinning situations.

\section{A. Green's function}

The vortex pinning problem considered here belongs to the class $D=3, n=2$, and the complex structure of the vortex lattice brings a number of modifications to the simple pinning model in Eq. (1). The Green's function for the vortex lattice (aligned along the $z$ axis) is in fact nondiagonal and features anisotropic and dispersive elastic moduli; focusing the discussion to isotropic superconductors and writing $\mathbf{k}=\left(\mathbf{K}, k_{z}\right)$ with the transverse $(\mathbf{K})$ and longitudinal $\left(k_{z}\right)$ components of the reciprocal vector, it assumes the form [7]

$$
G_{\alpha \beta}(\mathbf{k})=\frac{\mathcal{P}_{\alpha \beta}^{\|}(\mathbf{K})}{c_{11}(\mathbf{k}) K^{2}+c_{44}(\mathbf{k}) k_{z}^{2}}+\frac{\mathcal{P}_{\alpha \beta}^{\perp}(\mathbf{K})}{c_{66} K^{2}+c_{44}(\mathbf{k}) k_{z}^{2}}
$$

with indices $\alpha, \beta \in 1,2$ and the projection operators $\mathcal{P}_{\alpha \beta}^{\|}(\mathbf{K})=K_{\alpha} K_{\beta} / K^{2}$ and $\mathcal{P}_{\alpha \beta}^{\perp}(\mathbf{K})=\delta_{\alpha \beta}-K_{\alpha} K_{\beta} / K^{2}$. The compression and tilt moduli $c_{11}(\mathbf{k}) \approx c_{44}(\mathbf{k}) \approx\left(B^{2} / 4 \pi\right)(1+$ $\left.\lambda^{2} k^{2}\right)^{-1}$ exhibit strong (isotropic) dispersion due to the longrange interaction between vortices; $c_{66}=B \Phi_{0} /(8 \pi \lambda)^{2}$ is the nondispersive shear modulus ( $\mathbf{B} \| \hat{\mathbf{z}}$ is the magnetic field induced in the bulk of the superconductor). The corresponding real-space Green's function is obtained via standard Fourier transformation,

$$
G_{\alpha \beta}(\boldsymbol{\rho})=\int_{K<K_{\mathrm{BZ}}} \frac{d^{2} \mathbf{K} d k_{z}}{(2 \pi)^{3}} G_{\alpha \beta}(\mathbf{k}) e^{i \mathbf{k} \cdot \boldsymbol{\rho}},
$$

with the integration over $K$ restricted to the Brillouin zone of the vortex lattice, $K_{\mathrm{BZ}} \approx \sqrt{4 \pi} / a_{0}$. Of key importance will be the on-site Green's function $G_{\alpha \beta}(\boldsymbol{\rho}=\mathbf{0})=G(\mathbf{0}) \delta_{\alpha \beta}$. The integration in Eq. (4) then is dominated by transverse momenta near the Brillouin zone boundary $K \sim K_{\mathrm{BZ}}$, and estimating the relevant longitudinal momentum by comparing the shear and tilt elastic energies $c_{66} K^{2} \sim c_{44}\left(K_{\mathrm{BZ}}\right) k_{z}^{2}$, we obtain the scaling result $G(\mathbf{0}) \sim 1 /\left[a_{0} \sqrt{c_{44}\left(K_{\mathrm{BZ}}\right) c_{66}}\right]$. The precise integration in Eq. (4) gives the result $[23,25,33]$

$$
G(\mathbf{0})^{-1}=\zeta\left(a_{0}^{2} / \lambda\right) \sqrt{c_{44} c_{66}},
$$

where we define $c_{44}(k=0) \equiv c_{44}$ and with a numerical factor $\zeta$ that depends on the chosen approximation for the elastic moduli.

To evaluate the spatial variations of the Green's function, we consider a simplified model of the vortex lattice elasticity: we drop the first term in Eq. (3) involving the large compression modulus $c_{11}(\mathbf{k})>c_{66}$ and replace the projection operator in the remaining term by $\delta_{\alpha \beta}$, such that $G_{\alpha \beta}(\rho)=$ $G(\rho) \delta_{\alpha \beta}$. Our diagonal response function $G[\rho=(\mathbf{R}, z)]$ is characterized by a sharp and structured peak around the origin and a smooth decay $\propto 1 / \tilde{\rho}$ at large distances $\tilde{\rho}>\lambda$, where $\tilde{\boldsymbol{\rho}}=\left(\mathbf{R}, \sqrt{c_{66} / c_{44}} z\right)$ is the properly scaled distance due to the anisotropic elasticity of the vortex lattice. Going beyond the diagonal approximation does not change our strong pairpinning results obtained below. Note that the function $G(\rho)$ provides us with the displacement field $u(\rho)=G(\rho) F$ due to a $\delta$ force $F \delta(\rho)$ at the origin.

We first evaluate the Green's function in the nondispersive regime (large distances $\rho$ ), with the dominant contributions to the integration in Eq. (4) originating from small momenta $\lambda^{2} k^{2} \lesssim 1$ such that $c_{44}(k) \approx c_{44}$. The anisotropy of the Green's function in Eq. (3) generates different decays along the directions longitudinal and transverse to the induced magnetic field, i.e., for $\rho=(\mathbf{0}, z)$ and $\rho=(\mathbf{R}, 0)$. To simplify the calculation, we remove this anisotropy by introducing the rescaled momentum vector $\mathbf{q}=\left(\mathbf{K}, \sqrt{c_{44} / c_{66}} k_{z}\right)$ with $c_{44} / c_{66}=16 \pi \lambda^{2} / a_{0}^{2}$, which leads to

$$
G(\tilde{\boldsymbol{\rho}}) \approx \frac{1}{\sqrt{c_{44} c_{66}}} \int \frac{d^{3} \mathbf{q}}{(2 \pi)^{3}} \frac{e^{i \mathbf{q} \cdot \tilde{\boldsymbol{\rho}}}}{q^{2}},
$$

with $\sqrt{c_{44} c_{66}}=\left(B^{2} / 16 \pi \sqrt{\pi}\right)\left(a_{0} / \lambda\right)$ and the rescaled distance $\tilde{\boldsymbol{\rho}}=\left(\mathbf{R}, \sqrt{c_{66} / c_{44}} z\right)$. Integrating over the momenta $\mathbf{q}$ yields $G(\tilde{\rho})=1 /\left(4 \pi \sqrt{c_{44} c_{66}} \tilde{\rho}\right)$ and the reverse 


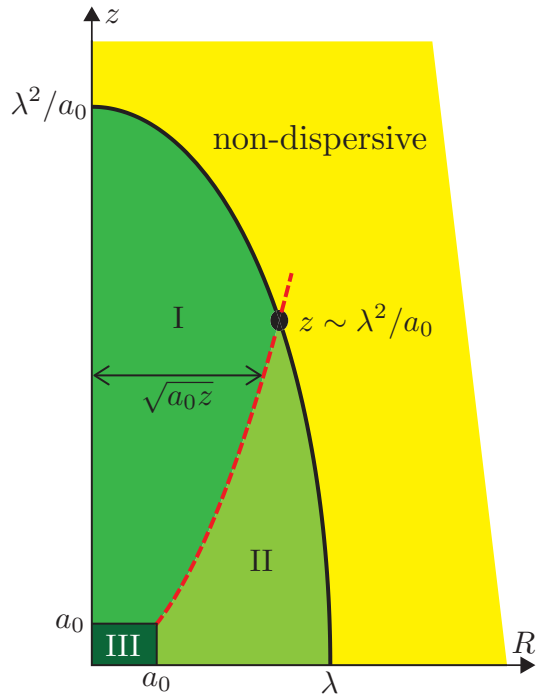

FIG. 2. Different domains analyzed in the evaluation of the scaled Green's function $g(\mathbf{R}, z)=G(\mathbf{R}, z) / G(\mathbf{0})$. In the nondispersive region (yellow) outside the ellipse $\tilde{\rho}^{2}=R^{2}+\left(a_{0}^{2} / 16 \pi \lambda^{2}\right) z^{2} \approx$ $\lambda^{2}$ the Green's function decays $\propto 1 / \tilde{\rho}$; see Eq. (7). The value of the Green's function on the ellipse boundary is $g(\tilde{\rho} \sim \lambda) \sim a_{0}^{2} / \lambda^{2}$. Inside the ellipse (green), we find several regions characterized by different scaling results. For $a_{0} \lesssim z \lesssim \lambda^{2} / a_{0}$, the Green's function reads $g(\mathbf{R}, z) \sim\left(a_{0} / z\right) e^{-\sqrt{\pi} R^{2} / a_{0} z}+a_{0}^{2} / \lambda^{2} ;$ starting out at $g(\mathbf{0}, z) \sim$ $a_{0} / z$, it decays exponentially fast along $R$ on the scale $R \sim \sqrt{a_{0} z}$ (region I, green) before saturating (ignoring slow logarithmic variations) at $g \sim a_{0}^{2} / \lambda^{2}$ (region II, light green). For small longitudinal coordinates $z \lesssim a_{0}$, the Green's function evaluated on the $z$ axis is $g(\mathbf{0}, z) \approx 1-z / a_{h}$, with $a_{h} \sim a_{0}\left[\ln \left(a_{0} / \xi\right)\right]^{1 / 2}$ the healing length, and its decay along the transverse coordinate $R$ is governed by the same scale $\sim a_{h}$ (region III, dark green). For $z \lesssim a_{0}, R \gtrsim a_{0}$, the Green's function again saturates at the value $g(\mathbf{R}, z) \sim a_{0}^{2} / \lambda^{2}$. Increasing $z$ at fixed $R$ within the interval $a_{0}<R<\lambda$, the ratio $g$ first increases and goes over a maximum when $z$ reaches the value $R^{2} / a_{0}$ (red dashes); this feature produces a distinct ridge in the peak region of $g$.

transformation $\tilde{\rho} \rightarrow \rho=(\mathbf{R}, z)$ provides us with the result

$$
G(R, z) \approx \frac{1 / 4 \pi \sqrt{c_{44} c_{66}}}{\sqrt{R^{2}+\left(a_{0}^{2} / 16 \pi \lambda^{2}\right) z^{2}}} .
$$

Equation (7) describes the situation where the dispersion in the tilt modulus can be neglected, which is the case at large distances $R^{2}+\left(a_{0}^{2} / 16 \pi \lambda^{2}\right) z^{2} \gtrsim \lambda^{2}$; see the yellow region in Fig. 2; on the inner boundary (an ellipsoid with extensions $R \sim \lambda$ and $\left.z \sim \lambda^{2} / a_{0}\right)$, the Green's function assumes a constant value $G \sim\left(a_{0}^{2} / \lambda^{2}\right) G(\mathbf{0})$ and decays $\propto 1 / \tilde{\rho}$ farther out; see Eq. (7). Indeed, in order to drop the dispersion in $c_{44}(k)$, we require the $q$ integral in (6) to be cut by a large distance $\tilde{\rho}$ (rather than the Brillouin zone), $q \lesssim 1 / \tilde{\rho}$, at values where $q \lambda<1$ (rendering dispersion irrelevant), implying that $\tilde{\rho}>\lambda$.

The evaluation of the Green's function at locations inside the ellipsoid requires proper integration both over small $\left(k \lesssim \lambda^{-1}\right)$ and large $\left(k \gtrsim \lambda^{-1}\right)$ momenta; the full calculation is presented in Appendix A. For longitudinal distances $z \gtrsim a_{0}$

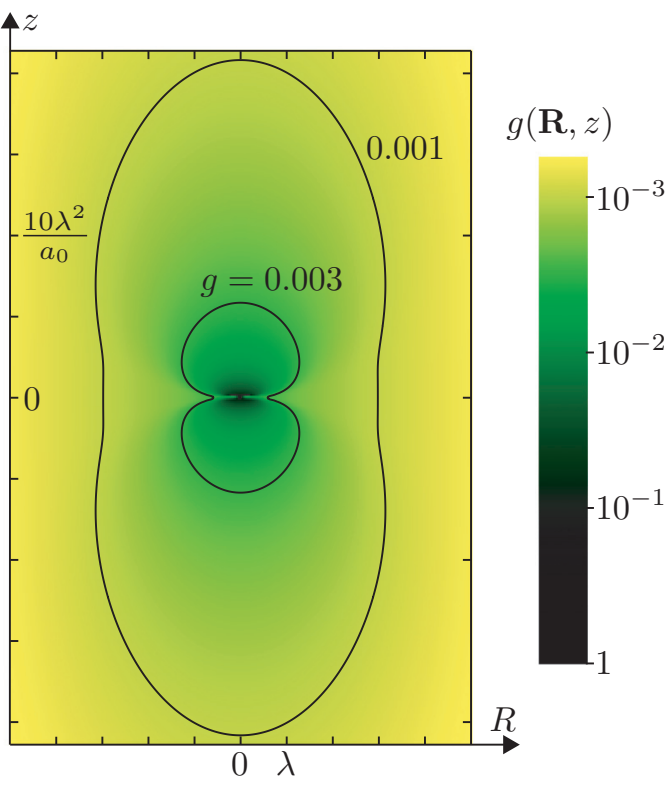

FIG. 3. Color plot of the rescaled Green's function $g(x=$ $R, 0, z)=G(R, 0, z) / G(\mathbf{0})$ evaluated for $\lambda=10 a_{0}$; axes are not to scale. The dark-green peak in the center saturates to unity over a region $\sim a_{0}^{2}$; at large distances $\tilde{\rho}=\sqrt{R^{2}+\left(a_{0}^{2} / 16 \pi \lambda^{2}\right) z^{2}}>$ $\lambda$, a smooth decay $\propto 1 / \tilde{\rho}$ is observed (yellow). The peak at small distances (green) exhibits a dumbbell shape and gives way to a smoothly decaying background of elliptical shape at large distances (yellow); the two contours with $g=$ 0.003 and $g=0.001$ illustrate this change of shape from a dumbbell to an elliptical form. Figure 11 in Appendix A shows the detailed contour plot near the center of the structured peak, including the position of the ridge.

and arbitrary $R$, we find the interpolation formula

$$
\begin{aligned}
G(\mathbf{R}, z) & \approx \frac{\lambda / \sqrt{4 \pi}}{a_{0} z \sqrt{c_{44} c_{66}}} e^{-\sqrt{\pi} R^{2} / a_{0} z} \\
& +\frac{1 / 16 \pi}{\lambda \sqrt{c_{44} c_{66}}}\left[1-2 \gamma+\ln \frac{16 \lambda^{2}}{R^{2}+a_{0} z / e^{\gamma} \sqrt{\pi}}\right]
\end{aligned}
$$

with $\gamma \approx 0.577$ the Euler-Mascheroni constant. This result provides us with various scaling regimes for the Green's function, as illustrated in Fig. 2. First, fixing $R=0$ and going away from the origin along the longitudinal direction, the rescaled Green's function decays as $G(\mathbf{0}, z) \sim\left(a_{0} / z\right) G(\mathbf{0})$; the result in Eq. (8) matches the nondispersive expression (7) at the crossover $z \sim \lambda^{2} / a_{0}$.

Increasing $R$ for $z<\lambda^{2} / a_{0}$, the Green's function is dominated by the first term in Eq. (8) that describes a Gaussian with height $G(\mathbf{R}=\mathbf{0}, z) \sim\left(a_{0} / z\right) G(\mathbf{0})$ and of width $R \sim \sqrt{a_{0} z}$; with decreasing $z$ this Gaussian peak becomes higher and narrows down to produce a dumbbell shape peak; see region I in the schematic Fig. 2 and the neck in the contour $g=0.003$ in Fig. 3. Increasing $R$ beyond $\sim \sqrt{a_{0} z}$, we enter region II in Fig. 2 where the second term in Eq. (8) dominates, interpolating smoothly between the peak and the nondispersive result Eq. (7). This smooth interpolation through region II is of order $G(\mathbf{R}, z) \sim\left(a_{0} / \lambda\right)^{2} G(\mathbf{0})$, with logarithmic corrections that become large at small values of $z$ where the narrow 
dumbbell peak at the origin decays more rapidly. Note that beyond the point $z \sim \lambda^{2} / a_{0}$ where the decay length $R \sim \sqrt{a_{0} z}$ meets the ellipsoidal shell, the Green's function for $\mathbf{R}=\mathbf{0}$ already assumes a value $G\left(\mathbf{0}, z \sim \lambda^{2} / a_{0}\right) \sim\left(a_{0}^{2} / \lambda^{2}\right) G(\mathbf{0})$ and no substantial variations with $R$ are seen within region I.

Increasing instead the longitudinal distance $z$ at fixed $R<$ $\lambda$, the Green's function first remains flat (region II), then steeply increases $\propto e^{-\sqrt{\pi} R^{2} / a_{0} z}$ upon entering the peak region I at $z \sim \pm R^{2} / a_{0}$, and then decreases smoothly $\propto 1 / z$, thus defining a maximum at $z \sim \pm R^{2} / a_{0}$. The resulting ridges located at the edges of the Gaussian peak are another manifestation of the dumbbell structure of the peak in $G(\mathbf{R}, z)$; see Figs. 3 and 11.

The discussion has to be further refined in the regime of small $z \lesssim a_{0}$. As $z \rightarrow 0$, the first term in Eq. (8) diverges for $R=0$ and vanishes for $R>0$, formally approaching the 2D delta function $\propto \delta^{2}\left(\mathbf{R} / a_{0}\right)$. In reality, accounting for the $\mathbf{q}$ cutoff at the Brillouin zone boundary in Eq. (4) provides us with the finite result for the on-site Green's function $G(\mathbf{R}=$ $\mathbf{0}, z=0)$. An expansion in the longitudinal direction for small $z \lesssim a_{0}$ then gives [34]

$$
G(\mathbf{0}, z) \approx\left(1-|z| / a_{h}\right) G(\mathbf{0}),
$$

with the healing length $a_{h} \sim a_{0}\left[\ln \left(a_{0} / \xi\right)\right]^{1 / 2}$.

The decay length in the transverse direction at small $z$ is affected by the single-vortex elasticity that becomes relevant near the Brillouin zone boundary [7,35]. Replacing the tilt modulus by $c_{44}(k) \rightarrow c_{44}(k)+\left(\varepsilon_{0} / a_{0}^{2}\right) \ln \left(a_{0} / \xi\right)$ then entails a saturation of the decay scale $R \sim \sqrt{a_{0} z}$ in Eq. (8) at $R \sim$ $a_{h} \sim a_{0}$ [we ignore a factor $\ln \left(a_{0} / \xi\right)$ in the scaling estimates] for $z \lesssim a_{0}$ (region III). For $R \gtrsim a_{h}$, we again cross over to region II where the Green's function assumes the constant value $\sim a_{0}^{2} / \lambda^{2}$, up to slow logarithmic corrections.

The above analysis has been carried out for a simplified diagonal expression $G_{\alpha \beta}=G \delta_{\alpha \beta}$. In a further step, one may replace the identity matrix $\delta_{\alpha \beta}$ by the full transverse projector $P_{\alpha \beta}^{\perp}(\mathbf{K})$; see Eq. (3). Focusing on the nondispersive regime, the $\mathbf{q}$ integral in Eq. (6) picks up an additional angular dependence on the geometry of the problem. For the component $G_{x x}$ evaluated in the $x z$-plane, we obtain the asymptotic dependence

$$
G_{x x}(R, 0, z) \approx \frac{1}{4 \pi \sqrt{c_{44} c_{66}}} \frac{\sqrt{R^{2}+\tilde{z}^{2}}-\tilde{z}}{R^{2}},
$$

where $\tilde{z}=\left(a_{0} / 4 \sqrt{\pi} \lambda\right) z$ is the scaled longitudinal length. The result (10) then exhibits a modified anisotropy at large distances: the simple scaling $G \propto 1 / \sqrt{R^{2}+\tilde{z}^{2}}$ in the expression (7) is replaced with $G_{x x} \propto 1 / 2 \tilde{z}$ when $\tilde{z} \gg R$ and $G_{x x} \propto$ $1 / R$ at large $R \gg \tilde{z}$. Finally, while $G_{x y}=0$, we find that $G_{y y}(R, 0, z)=\left(\tilde{z} / \sqrt{R^{2}+\tilde{z}^{2}}\right) G_{x x}$. Note that $G_{x x}+G_{y y}=G$, as expected.

Having analyzed the elastic component in the pinning problem, we now turn to the discussion of the pinning potential $\varepsilon_{\text {pin }}(\boldsymbol{\rho}, \mathbf{u})$ in Eq. (1) for the cases of strong pinning, weak-collective pinning, and the pinning by rare clusters. Note that the smallest transverse scale $R$ in the context of elasticity is the separation $a_{0}$ between vortices, while separations between defects as discussed below are considered small when $R$ reaches the effective size $\xi$ of defects. Hence, small lengths $R$ take a different meaning when talking about the vortex lattice (elasticity) or the pinning landscape.

\section{B. Strong pinning}

We consider a lattice of flux lines or vortices aligned with the $z$ axis and described by the unperturbed vortex core positions $\mathbf{R}_{\mu} \in \mathbb{R}^{2}$. The pinning force acts on the vortex cores and the pinning energy can be expressed in the form [with $\rho=(\mathbf{R}, z)]$

$$
\varepsilon_{\text {pin }}(\boldsymbol{\rho}, \mathbf{u})=\sum_{\mu} \delta^{(2)}\left(\mathbf{R}-\mathbf{R}_{\mu}\right) \varepsilon_{\text {pin }}^{\mu}\left[z, \mathbf{u}_{\mu}(z)\right]
$$

with $\varepsilon_{\text {pin }}^{\mu}\left[z, \mathbf{u}_{\mu}(z)\right]$ the random pinning potential acting on the $\mu$ th vortex line,

$$
\varepsilon_{\text {pin }}^{\mu}\left[z, \mathbf{u}_{\mu}(z)\right]=\int d^{2} \mathbf{R} U_{\text {pin }}(\mathbf{R}, z) p\left[\mathbf{R}-\mathbf{R}_{\mu}-\mathbf{u}_{\mu}(z)\right] .
$$

Here $U_{\text {pin }}(\mathbf{R}, z)$ denotes the disorder potential generated by the material defects; assuming pinning due to pointlike defects located at $\mathbf{r}_{i}=\left(\mathbf{R}_{i}, z_{i}\right)$, each with identical pinning energy $e_{p}$, the disorder potential takes the form

$$
U_{\text {pin }}(\mathbf{R}, z)=-\sum_{i} e_{p} \delta^{2}\left(\mathbf{R}-\mathbf{R}_{i}\right) \delta\left(z-z_{i}\right) .
$$

The factor $p(\mathbf{R})$ in Eq. (12) describes the vortex form factor, e.g., for a $\delta T_{c}$-type pinning mechanism [7], it reads $p(R)=$ $1-|\psi(R)|^{2}$, with $\psi(R)$ the superconducting order parameter of the single-vortex solution to the Ginzburg-Landau equations. The simple Ansatz [36,37] $|\psi(R)|=R /\left(R^{2}+2 \xi^{2}\right)^{1 / 2}$ provides us with Lorentzian shape for the form factor, $p(R)=$ $1 /\left(1+R^{2} / 2 \xi^{2}\right)$.

Combining Eqs. (12) and (13), we express the random pinning potential as

$$
\varepsilon_{\mathrm{pin}}^{\mu}\left[z, \mathbf{u}_{\mu}(z)\right]=\sum_{i} e_{p}\left[\mathbf{R}_{i}-\mathbf{R}_{\mu}-\mathbf{u}_{\mu}(z)\right] \delta\left(z-z_{i}\right),
$$

with $e_{p}(\mathbf{R})=-e_{p} p(\mathbf{R})$ the pinning potential due to a single defect; note that $e_{p}(\mathbf{R})$ is maximally negative for $\mathbf{R}=0$, i.e., pinning is maximal when the defect position $\mathbf{R}_{i}$ coincides with the perturbed vortex position $\mathbf{R}_{\mu}+\mathbf{u}_{\mu}(z)$. Substituting this result into Eqs. (2) and (11), we arrive at the equation for the displacement of the $v$ th vortex in the form

$$
\begin{aligned}
\mathbf{u}_{v}(z) & \equiv \mathbf{u}\left(\mathbf{R}_{v}, z\right) \\
& =\sum_{\mu, i} G\left(\mathbf{R}_{v}-\mathbf{R}_{\mu}, z-z_{i}\right) \mathbf{f}_{p}\left[\mathbf{R}_{\mu}+\mathbf{u}_{\mu}\left(z_{i}\right)-\mathbf{R}_{i}\right]
\end{aligned}
$$

with the pinning force

$$
\mathbf{f}_{p}(\mathbf{R})=-\nabla_{\mathbf{R}} e_{p}(\mathbf{R})=-\frac{e_{p}}{\xi} \frac{\mathbf{R} / \xi}{\left(1+R^{2} / 2 \xi^{2}\right)^{2}}
$$

acting in the direction transverse to the field. The last relation above applies for the Lorentzian-shaped potential.

In Eq. (15), we sum over all interactions between defects and vortices. In practice, we assume that no more than a single vortex can be pinned by an impurity and neglect interactions of vortices with defects far away from the vortex core, $\mid \mathbf{R}_{\mu}+$ $\mathbf{u}_{\mu}\left(z_{i}\right)-\mathbf{R}_{i} \mid \gg \xi$. The sum over the vortex index $\mu$ is then restricted to a single index $\mu(i)$ denoting the vortex closest to 
the impurity $i$. The relation (15) then allows to evaluate the displacement $\mathbf{u}_{\mu(i)}$ of the vortex $\mu$ pinned to the defect $i$ at the position $z_{i}$; this is nothing but the vortex tip displacement of the $\mu(i)$ th vortex,

$$
\begin{aligned}
\mathbf{u}_{i} \equiv \mathbf{u}_{\mu(i)}\left(z_{i}\right)= & \sum_{j} G\left(\mathbf{R}_{\mu(i)}-\mathbf{R}_{\mu(j)}, z_{i}-z_{j}\right) \\
& \times \mathbf{f}_{p}\left(\mathbf{R}_{\mu(j)}+\mathbf{u}_{j}-\mathbf{R}_{j}\right) .
\end{aligned}
$$

The set of equations (17) represents a system of $N$ coupled nonlinear equations for the displacements $\mathbf{u}_{i}$, with $N$ the total number of defects.

Within the strong pinning paradigm, we assume that defects act independently, allowing for a further simplification of Eq. (17) where the displacement $\mathbf{u}_{i}$ is ascribed exclusively to the action of the defect $i$; the summation over $j$ in Eq. (17) then reduces to the term $j=i$, i.e., we neglect the force exerted by distant defects $j \neq i$ on vortices $\mu(j)$ that contributes to the displacement $\mathbf{u}_{i}$ via the nonlocal Green's function $G\left(\mathbf{R}_{\mu(i)}-\mathbf{R}_{\mu(j)}, z_{i}-z_{j}\right)$. It is exactly this simplification that will be dropped later on when considering strong pinning by pairs. The system of equations (17) then reduces to $N$ independent equations

$$
\mathbf{u}_{i} \approx \mathbf{f}_{p}\left(\mathbf{R}_{\mu(i)}+\mathbf{u}_{i}-\mathbf{R}_{i}\right) / \bar{C}
$$

with the effective vortex-lattice elasticity defined by $\bar{C}=$ $1 / G(\mathbf{0})$; see Eq. (5).

The resulting pinning force density is obtained by summing the forces from all pinning sites. Note that the solution $\mathbf{u}_{i}$ in Eq. (18) depends only on the distance of the vortex from the pinning defect $\mathbf{x}_{i}=\mathbf{R}_{\mu(i)}-\mathbf{R}_{i}$. The average pinning force density is thus $\mathbf{F}_{\text {pin }}=n_{p}\left\langle\mathbf{f}_{p}[\mathbf{x}+\mathbf{u}(\mathbf{x})]\right\rangle_{\mathbf{x}}$, where $n_{p}$ denotes the density of impurities and the average is taken with respect to the possible position vectors $\mathbf{x}$; assuming a uniform distribution of relative distances $\mathbf{x}$, the average then corresponds to a simple integration over $\mathbf{x}$.

It turns out that the pinning force can be expressed as the gradient of the total pinning energy, $\mathbf{f}_{p}[\mathbf{x}+\mathbf{u}(\mathbf{x})]=$ $-\nabla_{\mathbf{x}} e_{\text {pin }}(\mathbf{x})$, with $e_{\text {pin }}(\mathbf{x})$ involving pinning and elastic terms,

$$
e_{\text {pin }}(\mathbf{x})=e_{p}[\mathbf{x}+\mathbf{u}(\mathbf{x})]+\frac{1}{2} \bar{C} \mathbf{u}(\mathbf{x})^{2} .
$$

If the solution $\mathbf{u}(\mathbf{x})$ to the on-site equation (18) is unique, implying a continuous evolution with $\mathbf{x}$, the average pinning force density vanishes, as follows from a simple integration of $\mathbf{f}_{p}[\mathbf{x}+\mathbf{u}(\mathbf{x})]$ over $\mathbf{x}$,

$$
\mathbf{F}_{\text {pin }}=-n_{p} \int \frac{d^{2} x}{a_{0}^{2}} \nabla_{\mathbf{x}} e_{\text {pin }}(\mathbf{x})=0 .
$$

The single-defect Ansatz is thus meaningful only in the strong pinning regime where the solution for the on-site displacement is nonunique. In this case, different values (branches) for the total pinning energy $e_{\text {pin }}(\mathbf{x})$ describe pinned and unpinned vortex states; see Fig. 4. Proper averaging accounts for the occupation of these branches, which is unsymmetric, resulting in a nonvanishing average pinning force density. We perform this analysis for a radially symmetric potential with a force $\mathbf{f}_{p}(\mathbf{r})=\hat{\mathbf{r}} f_{p}(r)$.

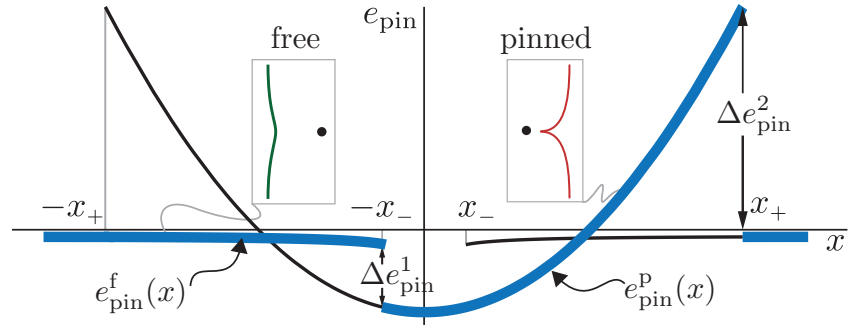

FIG. 4. Sketch of a typical large- $\kappa$ energy profile $e_{\text {pin }}(x)$ with multiple branches involving an approximately parabolic pinned branch and nearly flat free branches. The branch occupation (denoted by thick blue lines) changes at the points $-x_{-}$and $x_{+}$that are associated with the pinning and depinning processes for a vortex passing the defect. The sum of the associated energy jumps $\Delta e_{\mathrm{pin}}^{1}$ and $\Delta e_{\text {pin }}^{2}$ provide a quantitative result for the pinning force density.

The condition for the appearance of multiple solutions is provided by the Labusch criterion $[4,19,28]$

$$
\kappa=\frac{\max f_{p}^{\prime}(r)}{\bar{C}}>1
$$

[we note that $\max f_{p}^{\prime}(r)=f_{p}^{\prime}\left(r_{m}\right)$ with the inflection point $r_{m}$ obtained from $\left.f_{p}^{\prime \prime}\left(r_{m}\right)=0\right]$. Furthermore, we assume that the vortices are driven in the positive $x$ direction, and we parametrize their trajectories $\mathbf{x}=(x, b)$ through the longitudinal vortex position $x$ and an impact parameter $b$ in the transverse direction (the distinction between the "longitudinal" field direction along $z$ and the "longitudinal" direction of motion along $x$ should be clear from the context). The resulting pinning force averaged over positions $\mathbf{x}$ then points in the negative $x$ direction and is evaluated in two steps: first, we perform an averaging over $x$ at vanishing impact parameter $b=0$, and then we average over contributions from vortex trajectories with finite impact parameters $b \neq 0$.

For the case of a vanishing impact parameter $b=0$, Eq. (18) can be reduced to one dimension, $\bar{C} u=f_{p}(x+u)$ (we have dropped the index $i$ ). This is equivalent to minimizing the total pinning energy $e_{\text {pin }}(x, u)=e_{p}(x+u)+\frac{1}{2} \bar{C} u^{2}$ with respect to $u$. Provided $x$ falls into the bistability region, $|x| \in\left[x_{-}, x_{+}\right]$, there exist multiple solutions $u_{\mathrm{f}}(x), u_{\mathrm{p}}(x)$ for the vortex tip displacement (denoting free and pinned vortex states) $[23,30,33]$. Substituting these solutions to the total pinning energy provides multiple branches $e_{\text {pin }}^{\mathrm{f}, \mathrm{p}}(x) \equiv$ $e_{\mathrm{pin}}\left[x, u_{\mathrm{f}, \mathrm{p}}(x)\right]$; see Fig. 4 . When going from large negative to large positive $x$, the branch occupation first undergoes a transition from the free to the pinned branch at the pinning point $-x_{-}$and then another transition from the pinned to the free branch at the depinning point $x_{+}$. Averaging the pinning force $f_{p}\left[x+u_{o}(x)\right]$ over the occupied branches (as marked by the index $o \in\{\mathrm{f}, \mathrm{p}\}$ ), the resulting integral over $x$ can be expressed as [see Eq. (20) and [23,27,33]],

$$
\left\langle f_{p}\left[x+u_{o}(x)\right]\right\rangle_{x}=-\frac{\Delta e_{\mathrm{pin}}^{1}+\Delta e_{\mathrm{pin}}^{2}}{a_{0}},
$$

with the jumps in energy $\Delta e_{\mathrm{pin}}^{1}=\left[e_{\mathrm{pin}}^{\mathrm{f}}-e_{\mathrm{pin}}^{\mathrm{p}}\right]_{x=-x_{-}}$and $\Delta e_{\mathrm{pin}}^{2}=\left[e_{\mathrm{pin}}^{\mathrm{p}}-e_{\mathrm{pin}}^{\mathrm{f}}\right]_{x=x_{+}}$occurring at the pinning $\left(-x_{-}\right)$and depinning $\left(x_{+}\right)$points, respectively. 
The result (22) remains unchanged even for a nonvanishing impact parameter $b \neq 0[23,27]$, provided the vortex passes the defect within the pinning distance $y_{p}$ along the $y$ direction; for the radially symmetric case, it turns out that $y_{p}=x_{-}$and hence pinning occurs for impacts with $|b|<x_{-}$. For $|b|>x_{-}$, the pinning forces are small and multiple branches no longer exist, implying a vanishing average over $x[23,27]$. Finally, averaging the result (22) over $y$ contributes a factor $2 x_{-} / a_{0}$ and thus

$$
\left\langle\mathbf{f}_{p}[\mathbf{x}+\mathbf{u}(\mathbf{x})]\right\rangle_{\mathbf{x}}=\left(-\mathbf{e}_{x}\right) \frac{2 x_{-}}{a_{0}} \frac{\Delta e_{\mathrm{pin}}^{1}+\Delta e_{\mathrm{pin}}^{2}}{a_{0}},
$$

with $-\mathbf{e}_{x}$ denoting the unit vector pointing in the negative $x$ direction.

Equation (23) assumes different scaling forms for the regime of very strong pinning $\kappa \gg 1$ and for moderately strong pinning $\kappa-1 \ll 1$. In the first case, the jump sizes are related to the pinning potential depth via $\Delta e_{\text {pin }}^{1} \sim e_{p}$ and $\Delta e_{\text {pin }}^{2} \sim \kappa e_{p}[23,27]$, together providing the estimate for the magnitude of the position-averaged pinning force $\left\langle f_{p}\right\rangle_{\mathbf{x}} \sim$ $\left(\kappa \xi^{2} / a_{0}^{2}\right) f_{p}$ and a pinning force density

$$
F_{\text {pin }} \sim \frac{\kappa \xi^{2}}{a_{0}^{2}} n_{p} f_{p}=\frac{S_{\text {trap }}}{a_{0}^{2}} n_{p} f_{p} .
$$

This result is interpreted as a pinning force $f_{p} \sim e_{p} / \xi$ [see Eq. (16)] due to a single defect exerted within the trapping area $[28,31] S_{\text {trap }}=2 y_{p}\left(x_{+}+x_{-}\right) \sim \kappa \xi^{2} ; S_{\text {trap }} / a_{0}^{2}$ denotes the fraction of area occupied by trapped vortices.

For moderately strong pinning with $\kappa$ close to unity (that is particularly relevant for the pinning by rare events), the energy jumps are evaluated by expanding the pinning force around the inflection point at $r_{m}, f_{p}^{\prime \prime}\left(r_{m}\right)=0$, where $f_{p}^{\prime}\left(r_{m}\right)=\kappa \bar{C}$ is maximally positive [27-29,33],

$$
f_{p}\left(r_{m}+\delta r\right) \approx f_{p}\left(r_{m}\right)+\kappa \bar{C} \delta r+\frac{1}{6} f_{p}^{\prime \prime \prime}\left(r_{m}\right) \delta r^{3} .
$$

In this situation, both jumps are identical and given by the expression $[27,28]$ [note that $\left.f_{p}^{\prime \prime \prime}\left(r_{m}\right)<0\right]$

$$
\Delta e_{\mathrm{pin}}^{1}=\Delta e_{\mathrm{pin}}^{2}=\frac{9 \bar{C}^{2}}{2\left[-f_{p}^{\prime \prime \prime}\left(r_{m}\right)\right]}(\kappa-1)^{2} .
$$

Using the scaling formulas $\bar{C}=f_{p}^{\prime}\left(r_{m}\right) / \kappa \sim f_{p} \xi$ [provided $\kappa \sim O(1)], f_{p}^{\prime \prime \prime}\left(r_{m}\right) \sim f_{p} / \xi^{3}$ in Eq. (26) then gives

$$
\left\langle f_{p}\right\rangle_{\mathbf{x}} \sim \frac{\xi^{2}}{a_{0}^{2}} f_{p}(\kappa-1)^{2} .
$$

The pinning force density follows trivially,

$$
F_{\text {pin }} \sim \frac{\xi^{2}}{a_{0}^{2}}(\kappa-1)^{2} n_{p} f_{p},
$$

and vanishes at the Labusch point $\kappa=1$, in accordance with the strong pinning criterion (21).

\section{Weak-collective pinning}

When pinning is weak, $\kappa<1$, individual defects fail to produce multivalued solutions for the vortex displacement and the mean pinning force in Eq. (20) vanishes. Pinning then arises through the random action of defects within the collective pinning volume $V_{c}$ defined as the region where the spatial fluctuations of the vortex displacement $\left\langle u^{2}(\boldsymbol{\rho})\right\rangle=\langle[\mathbf{u}(\boldsymbol{\rho})-$ $\mathbf{u}(\mathbf{0})]\rangle^{2}$ remains bounded by the pinning scale, $\left\langle u^{2}(\rho)\right\rangle \leqslant \xi^{2}$. The displacement correlation function can be systematically evaluated from Eq. (2) using the disorder-averaged correlator of the pinning energy density Eq. (11) [7,11],

$$
\left\langle\varepsilon_{\operatorname{pin}}(\boldsymbol{\rho}, \mathbf{u}) \varepsilon_{\operatorname{pin}}\left(\boldsymbol{\rho}^{\prime}, \mathbf{u}^{\prime}\right)\right\rangle=\frac{e_{p}^{2} n_{p}}{a_{0}^{2}} \delta^{3}\left(\boldsymbol{\rho}-\boldsymbol{\rho}^{\prime}\right) k\left(\mathbf{u}-\mathbf{u}^{\prime}\right),
$$

with the correlation function $k\left(\mathbf{u}-\mathbf{u}^{\prime}\right)=\int d^{2} \mathbf{R} p(\mathbf{R}-$ u) $p\left(\mathbf{R}-\mathbf{u}^{\prime}\right)$ related to the vortex form factor $p(R)$.

A qualitative estimate for the displacement correlator is provided by summing up distortions originating from all defects within a finite volume. In the vicinity of a reference defect characterized by the pinning force $f_{p} \sim e_{p} / \xi$, the distortion scale $u_{0}$ is given by the on-site Green's function, $u_{0} \sim$ $G(\mathbf{0})^{-1} f_{p}$. Expressing the on-site displacement through the effective vortex lattice stiffness $\bar{C}=G(\mathbf{0})^{-1}$ and estimating the Labusch parameter as $\kappa \sim f_{p} / \bar{C} \xi$ provides us with $u_{0} \sim$ $\kappa \xi$. Assuming small defect densities and hence large typical interdefect separations, the extension of the collective pinning volume falls into the nondispersive regime of the Green's function; see Eq. (7). Defects located a distance $\tilde{\rho}^{2}=R^{2}+$ $\left(a_{0}^{2} / 16 \pi \lambda^{2}\right) z^{2}$ away from the reference defect contribute with the displacement $u(\tilde{\rho}) \sim u_{0} G(\tilde{\boldsymbol{\rho}}) / G(\mathbf{0}) \sim u_{0}\left(a_{0}^{2} / \lambda \tilde{\rho}\right)$. Within the collective pinning volume $V_{c}=R_{c}^{2} L_{c} \sim\left(\lambda / a_{0}\right) R_{c}^{3}$, these displacements add up with a random sign, as the forces from different defects are randomly directed; furthermore, only the fraction $\xi^{2} / a_{0}^{2}$ of defects that reside inside the vortex cores are directly attacking the vortices, resulting in a total squared displacement $\left\langle u^{2}\left(R_{c}\right)\right\rangle \sim\left(\kappa \xi a_{0}^{2} / \lambda R_{c}\right)^{2}\left(\xi^{2} / a_{0}^{2}\right) n_{p} V_{c}$ on the scale $R_{c}$. Finally, the condition $\left\langle u^{2}\left(R_{c}\right)\right\rangle \sim \xi^{2}$ provides us with the collective pinning radius

$$
R_{c} \sim \lambda \frac{1}{\kappa^{2} n_{p} \xi^{2} a_{0}} .
$$

For small defect densities, as specified by the condition $\kappa^{2} n_{p} \xi^{2} a_{0} \ll 1$, the pinning radius $R_{c} \gg \lambda$ indeed falls into the nondispersive regime (note that $\kappa \lesssim 1$ ). Finally, summing up the random force contributions due to the active defects within the bundle volume $V_{c}=\left(\lambda / a_{0}\right) R_{c}^{3}, F_{\text {coll }} \sim$ $\left[f_{p}^{2} n_{p}\left(\xi^{2} / a_{0}^{2}\right) V_{c}\right]^{1 / 2} / V_{c}$, we find the collective pinning force density

$$
F_{\text {coll }} \sim \frac{\xi^{2}}{\lambda^{2}} \kappa^{3}\left(n_{p} a_{0} \xi^{2}\right) n_{p} f_{p}
$$

\section{Pinning by rare events}

The collective pinning scenario described above sums up small competing contributions to the vortex lattice distortions arising from typical fluctuations in the defect distribution, involving defects that lie far away from each other within the collective pinning volume. However, it does not account for the presence of rare clusters, where two (or more) weak defects act cooperatively, giving rise to an effectively strong pinning center; the latter then is supposed to produce a distortion exceeding the scale $\xi$ of the pinning potential. In looking for promising candidate pairs, we consider Eqs. (7) 
and (8) that describe the decay of the Green's function; these imply that the vortex displacement is substantially suppressed beyond a distance $\sim a_{0}$ away from the defect. Hence, two weak defects with $\frac{1}{2}<\kappa<1$ can be combined into a strong pinning object characterized by $\kappa>1$ and producing a displacement $u$ of order $\xi$ only if they are at most a longitudinal distance $z \sim a_{0}$ apart and pinning the same vortex core, i.e., they are separated by at most $R \sim \xi$ in the transverse dimension. This consideration then provides us with the density $\left(n_{p} a_{0} \xi^{2}\right) n_{p}$ of strong pinning pairs. With only the fraction $\xi^{2} / a_{0}^{2}$ of those clusters being located within the vortex core area and each cluster exerting a pinning force $\sim f_{p}$, we arrive at the following estimate for the pinning force density due to defect pairs (with $1 / 2<\kappa<1$ still close to unity),

$$
F_{\text {clust }} \sim \frac{\xi^{2}}{a_{0}^{2}}\left(n_{p} a_{0} \xi^{2}\right) n_{p} f_{p} .
$$

Assuming a magnetic field sufficiently above $H_{c 1}$ such that $a_{0}<\lambda$, the pinning force due to such clusters dominates over the collective pinning contribution in Eq. (31) by a factor of $\left(\lambda / a_{0}\right)^{2}$.

This factor in fact arises due to the dispersion of the tilt elastic modulus; in order to trace its origin, we need to understand the explicit dependence of the quantities contributing to both pinning mechanisms on the elastic properties of the vortex lattice, a dispersive tilt modulus $c_{44}(k)$ and nondispersive shear modulus $c_{66}$. The collective pinning radius in Eq. (30) can be obtained by comparing the elastic energy $\mathcal{E}_{\text {el }}\left(V_{c}\right) \sim c_{66}\left(\xi / R_{c}\right)^{2} V_{c}$ and the pinning energy $\mathcal{E}_{\text {pin }}\left(V_{c}\right) \sim$ $\left[f_{p}^{2} n_{p}\left(\xi^{2} / a_{0}^{2}\right) V_{c}\right]^{1 / 2} \xi$ accumulated within the pinning domain of volume $V_{c}=R_{c}^{2} L_{c}$. Assuming a large collective pinning volume where the dispersion of the tilt modulus is not relevant, the extensions $R_{c}$ and $L_{c}$ in the longitudinal and the transverse directions are related via $c_{66}\left(\xi / R_{c}\right)^{2} \sim c_{44}\left(\xi / L_{c}\right)^{2}$, that provides us with (we write $\left.c_{44}(k=0) \equiv c_{44}\right)$

$$
R_{c} \sim \frac{c_{66}^{3 / 2} c_{44}^{1 / 2} a_{0}^{2}}{f_{p}^{2} n_{p}},
$$

and the pinning force density is estimated as

$$
F_{\text {coll }} \sim \frac{\mathcal{E}_{\text {el }}\left(V_{c}\right)}{V_{c} \xi} \sim \frac{\xi}{a_{0}^{4}} \frac{f_{p}^{4} n_{p}^{2}}{c_{66}^{2} c_{44}} .
$$

We replace a factor $f_{p}^{3}$ in (34) with $\kappa^{3}(\bar{C} \xi)^{3}$,

$$
F_{\text {coll }} \sim \frac{\xi^{4}}{a_{0}^{4}} n_{p}^{2} f_{p} \frac{\kappa^{3} \bar{C}^{3}}{c_{66}^{2} c_{44}},
$$

and using $\kappa \sim 1, \bar{C} \sim a_{0}\left[c_{44}\left(K_{\mathrm{BZ}}\right) c_{66}\right]^{1 / 2}$, and $\left[c_{44}\left(K_{\mathrm{BZ}}\right) /\right.$ $\left.c_{66}\right]^{1 / 2} \sim 1$, we obtain the desired result

$$
F_{\text {coll }} \sim \frac{c_{44}\left(K_{\mathrm{BZ}}\right)}{c_{44}} \frac{\xi^{2}}{a_{0}^{2}}\left(n_{p} a_{0} \xi^{2}\right) n_{p} f_{p} .
$$

On the other hand, our strong pinning pairs are small and the associated elastic scales $R_{p}$ and $L_{p}$ in the longitudinal and transverse directions are related by $c_{66}\left(u / R_{p}\right)^{2} \sim$ $c_{44}\left(K_{\mathrm{BZ}}\right)\left(u / L_{p}\right)^{2}$ with the short scale elasticity $c_{44}\left(K_{\mathrm{BZ}}\right)$; hence $L_{p} \sim a_{0} \sqrt{c_{44}\left(K_{\mathrm{BZ}}\right) / c_{66}} \sim a_{0}$, where we have chosen the smallest transverse scale $R_{p} \sim a_{0}$ of the lattice. The density of pairs then is given by $n_{p}\left(n_{p} \xi^{2} a_{0}\right)$ and the resulting pair-pinning force density is [assuming again $\kappa \sim O(1)$; cf. Eq. (32)]

$$
F_{\text {clust }} \sim \frac{\xi^{2}}{a_{0}^{2}}\left(n_{p} a_{0} \xi^{2}\right) n_{p} f_{p} .
$$

Finally, comparing the weak-collective and cluster-pinning force densities in Eqs. (36) and (37) provides us with

$$
\frac{F_{\text {clust }}}{F_{\text {coll }}} \sim \frac{c_{44}}{c_{44}\left(K_{\mathrm{BZ}}\right)} \sim \frac{\lambda^{2}}{a_{0}^{2}},
$$

which demonstrates that the cluster pinning dominates over the weak-collective pinning contributions due to the dispersion in the tilt modulus with its reduction $\propto\left(a_{0} / \lambda^{2}\right)$ at the Brillouin zone boundary.

The concept of pair pinning described above can be extended to larger clusters, pushing the domain of pinning by rare events further down to smaller values of $\kappa$. Within the interval $\kappa \in[1 / n, 1 /(n-1)], n \geqslant 2$ and integer, $n$ neighboring defects are required to form a strong pinning cluster with $n \kappa>1$; the density of such clusters is given by $\left(n_{p} a_{0} \xi^{2}\right)^{n-1} n_{p}$, and the resulting pinning force density becomes $F_{\text {clust }} \sim$ $\left(\xi / a_{0}\right)^{2}\left(n_{p} a_{0} \xi^{2}\right)^{n-1} n_{p} f_{p}$. However, for pinning strengths $\kappa \leqslant$ $1 / n$ with $n \approx 2+2\left[\ln \left(\lambda / a_{0}\right)\right] /\left[\ln \left(1 / n_{p} a_{0} \xi^{2}\right)\right]$, the collective pinning dominates; given a low density of defects such that $n_{p} a_{0} \xi^{2} \ll\left(a_{0} / \lambda\right)^{2}$, this crossover lies close to $n=2, \kappa=\frac{1}{2}$.

The idea of pinning due to rare events has been previously touched upon in the context of charge density wave pinning in high dimensions; see Ref. [32]. In this case, the disorderinduced distortions accumulated over a finite-sized domain are not sufficient to induce pinning. This can be easily seen by considering the elastic Green's function $G(\rho) \propto \rho^{2-D}$ in $D$ dimensions, yielding a total displacement accumulated within a pinning domain of size $R$ that scales as $\left\langle u^{2}(R)\right\rangle \sim R^{4-D}$; see Eq. (2). While for $D<4$, the accumulated displacement will eventually exceed the threshold required for the existence of bistabilities at large domain sizes $R$, this is not the case for dimensions $D \geqslant 4$. As noted by Fisher [32], this does not render the weak disorder irrelevant, since, although with exponentially small probability, one will always find rare domains with anomalously coherent pinning. The manifold is then pinned by such rare fluctuations rather than by the collective action of the disorder landscape. In our $D=3$ vortex lattice, the situation is somewhat different: for $D=3$, weak pinning is still active and competes with the pinning by rare events, which take the specific form of nearby defect pairs making up for a strong pinning object. The latter mechanism dominates for defect strengths $\frac{1}{2}<\kappa<1$ and a small density of defects. The dominance of pinning by such rare events is, however, not an inherent property of the pinning mechanism, but rather appears as a result of the specific, i.e., dispersive, elastic response of the vortex lattice.

\section{TWO-DEFECT PROBLEM}

We have seen in Sec. II B that the strong pinning paradigm assuming independent action of defects is meaningful only provided the Labusch parameter (21) satisfies $\kappa>1$; in this case, the single-defect Ansatz gives rise to multivalued solutions for the vortex displacement, which results in a finite 
averaged pinning force. Here we consider the range of pinning strength $\frac{1}{2}<\kappa<1$ and go a step beyond the single-defect Ansatz by considering pairs of defects. Given $N$ defects, of all possible $N(N-1) / 2$ pairings there will be a finite set of pairs that reach the strong pinning criterion, thus generating multivalued solutions of vortex states; given that defects are dilute, these strong pinning pairs will be dilute as well and hence act independently.

\section{A. Geometry}

To find the relevant pairs, we consider two defects labeled by $i=1,2$ at positions $\left(\mathbf{R}_{i}, z_{i}\right)$ and the associated vortices $\mathbf{R}_{\mu(i)}$ separated from the pins by $\mathbf{x}_{i}=\mathbf{R}_{\mu(i)}-\mathbf{R}_{i}$; the displacement fields $\mathbf{u}_{i}$ at $z_{i}$ are solutions of the coupled equations [see Eq. (17)],

$$
\begin{aligned}
\bar{C} \mathbf{u}_{1} & =\mathbf{f}_{p}\left(\mathbf{x}_{1}+\mathbf{u}_{1}\right)+g \mathbf{f}_{p}\left(\mathbf{x}_{2}+\mathbf{u}_{2}\right), \\
\bar{C} \mathbf{u}_{2} & =\mathbf{f}_{p}\left(\mathbf{x}_{2}+\mathbf{u}_{2}\right)+g \mathbf{f}_{p}\left(\mathbf{x}_{1}+\mathbf{u}_{1}\right) .
\end{aligned}
$$

The coupling $g \in(0,1]$ renormalizes the force at the site of the first impurity due to the action of the second impurity (and vice versa) and reads

$$
g=G\left(\mathbf{R}_{\mu(1)}-\mathbf{R}_{\mu(2)}, z_{1}-z_{2}\right) / G(\mathbf{0}) .
$$

While the impurity positions $\mathbf{R}_{i}$ and displacements $\mathbf{u}_{1,2}$ in (39) are continuous variables (with the small scale set by $\xi$ ), the vortex positions $\mathbf{R}_{\mu(1,2)}$ in (40) are restricted to the vortex lattice involving the scale $a_{0}$. A coupling $g$ of order unity implies that both impurities act with their maximal pinning force on the same vortex; typical separations of such defects are below $\xi$ in the transverse and below $a_{0}$ in the longitudinal direction. Hence, large couplings $g$ are associated with close defect pairs lying within a volume $a_{0} \xi^{2}$. Small couplings $g \ll 1$ refer to the situation where the impurities are separated far away from one another, of order several lattice constants $a_{0}$, typically; in this situation, the defects act on different vortices and their mutual effect on the vortex pair is small.

As in Sec. II B, we consider a driving force applied in the positive $x$ direction and assume that the vortex lattice structure is preserved; under application of the drive, the vortices are displaced from their initial positions $\mathbf{R}_{\mu(i)}^{0}$ by a constant shift of magnitude $X$ along $x$, i.e, $\mathbf{R}_{\mu(i)}=\mathbf{R}_{\mu(i)}^{0}+X \mathbf{e}_{x}$. It is convenient to reformulate the problem in terms of the mean vortex position $\mathbf{x}$ (relative to the defects)

$$
\begin{aligned}
\mathbf{x} & =\frac{1}{2}\left(\mathbf{x}_{1}+\mathbf{x}_{2}\right) \\
& =\frac{1}{2}\left(\mathbf{R}_{\mu(1)}^{0}+\mathbf{R}_{\mu(2)}^{0}\right)-\frac{1}{2}\left(\mathbf{R}_{1}+\mathbf{R}_{2}\right)+X \mathbf{e}_{x},
\end{aligned}
$$

and the mismatch vector $\boldsymbol{\Delta}$,

$$
\boldsymbol{\Delta}=\mathbf{x}_{1}-\mathbf{x}_{2}=\mathbf{R}_{\mu(1)}^{0}-\mathbf{R}_{1}-\left(\mathbf{R}_{\mu(2)}^{0}-\mathbf{R}_{2}\right) ;
$$

see Fig. 5. Note that the vortex positions $\mathbf{x}_{1}$ and $\mathbf{x}_{2}$ (relative to the defects) as well as the mismatch vector $\boldsymbol{\Delta}$ are restricted to the unit cell of the vortex lattice.

Pushing the vortex lattice a distance $X$ along the $x$ direction, the mean vortex position $\mathbf{x}$ is parametrized as $\mathbf{x}=$ $[x(X), b]$ with a fixed impact parameter $b$, while the vector $\Delta$ remains constant. Since $\mathbf{x}_{1}=\mathbf{x}+\boldsymbol{\Delta} / 2$ and $\mathbf{x}_{2}=\mathbf{x}-\boldsymbol{\Delta} / 2$ (see Fig. 5), the vector $\boldsymbol{\Delta}$ is interpreted as the mismatch in the pinning by the two defects. If $\boldsymbol{\Delta}=\mathbf{0}$, the defects are (a)

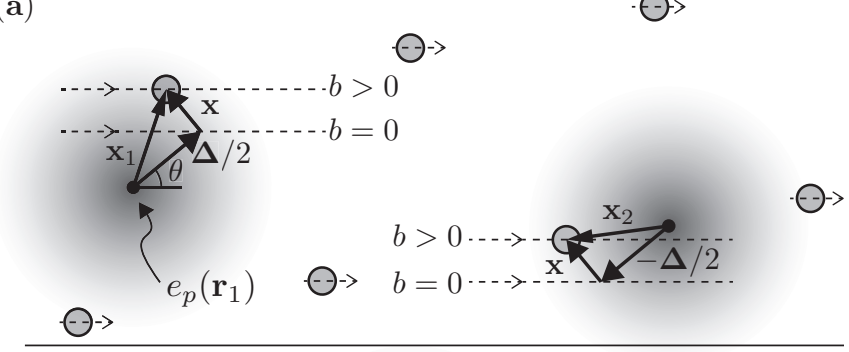

(b)

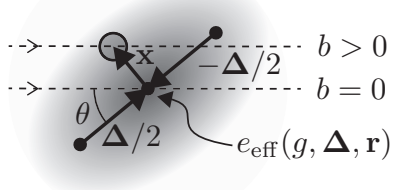

FIG. 5. Explanation of the mean vortex position $\mathbf{x}$ and the mismatch vector $\boldsymbol{\Delta}=(\Delta \cos \theta, \Delta \sin \theta)$ for a vortex lattice driven in the positive $x$ direction. (a) Two defects (black points) are pinning two vortices (gray circles) at the unperturbed relative (to the defects) positions $\mathbf{x}_{1}=\boldsymbol{\Delta} / 2+\mathbf{x}$ and $\mathbf{x}_{2}=-\boldsymbol{\Delta} / 2+\mathbf{x}$. The areas shaded in gray represent the defects' pinning potentials $e_{p}\left(\mathbf{r}_{i}\right)$ with $\mathbf{r}_{i}=\mathbf{x}_{i}+\mathbf{u}_{i}$, $i \in\{1,2\}$, the positions of the vortex tips displaced by $\mathbf{u}_{i}$ (not shown on the picture). The other vortices of the lattice are not affected by any of the defects but their asymptotic positions are co-moving with the vortex lattice. The pinning-force averaging is performed over the trajectories $\mathbf{x}=(x, b)$; shown is an example of a trajectory with impact parameter $b>0$. Shifting the whole lattice downwards produces the special trajectory of maximal symmetry characterized by $b=0$. In this case, both vortices are separated from the defect by the same transverse distance $|\Delta \sin \theta|$ (along $y$ ). When passing through the point $\mathbf{x}=0$, their position relative to the defect is given by opposite vectors $\Delta / 2$ and $-\Delta / 2$. (b) Reduction of the two-vortex problem to the pinning of an effective vortex at the position $\mathbf{x}=\frac{1}{2}\left(\mathbf{x}_{1}+\mathbf{x}_{2}\right)$ relative to an effective defect characterized by an anisotropic (or nonradial) pinning potential $e_{\text {eff }}(g, \boldsymbol{\Delta} ; \mathbf{r})$, with $\mathbf{r}=\mathbf{x}+\mathbf{u}$ and $\mathbf{u}=$ $\frac{1}{2}\left(\mathbf{u}_{1}+\mathbf{u}_{2}\right)$ the displacement of the effective vortex. For $b=0$ the effective vortex passes through the center of $e_{\text {eff }}(\mathbf{r})$.

perfectly synchronized: for any $X$, the position of both vortices relative to the defects is the same, $\mathbf{x}_{1}=\mathbf{x}_{2}$, the pinning forces acting on both vortices are identical, and pinning by the defect pair is maximal. For a finite $\boldsymbol{\Delta} \neq \mathbf{0}$, the two vortices are subject to different pinning forces, which reduces the total pinning strength. As shown in Fig. 5(b), the geometry can be reduced to one where an effective vortex at the position $\mathbf{x}$ impacts on an effective defect with a nonradial pinning potential $e_{\text {eff }}(g, \boldsymbol{\Delta} ; \mathbf{r})$, with $\mathbf{r}=\mathbf{x}+\mathbf{u}$ and $\mathbf{u}=\frac{1}{2}\left(\mathbf{u}_{1}+\mathbf{u}_{2}\right)$ the displacement of the effective vortex.

Figure 5 also clarifies the meaning of the head-on vortex trajectory $\mathbf{x}=[x(X), 0]$ with vanishing impact parameter $b$ in the context of the two-defect problem. When $\boldsymbol{\Delta} \neq \mathbf{0}$, it is not possible for both trajectories $\mathbf{x}_{1}, \mathbf{x}_{2}$ to simultaneously pass through the defect centers. The special value $b=0$ then describes the situation where both vortices are separated by the same transverse distance from the defects and the vortex trajectory passes through the special point $\mathbf{x}=0$ when the vortices are located at opposite positions $\Delta / 2,-\boldsymbol{\Delta} / 2$ with 
respect to the defects. Translated to the effective geometry, for $b=0$ the effective vortex passes through the center of $e_{\text {eff }}(\mathbf{r})$.

\section{B. Averaging}

Given the geometric layout of the strong pinning problem with two vortices and two defects, we have to find the associated pinning force density $F_{\text {pin }}$ by proper averaging. This averaging involves (1) the averaging over trajectories $\mathbf{x}=(x, b)$ of vortex pairs with fixed mismatch $\boldsymbol{\Delta}$ and fixed coupling $g$, (2) the averaging over all possible mismatch vectors $\boldsymbol{\Delta}$, and (3) the averaging over couplings $g$ in the pair pinning Eq. (39) that involves the relative distances between vortices $\mathbf{R}_{\mu(1)}-\mathbf{R}_{\mu(2)}$ and the distance in elevation $z_{1}-z_{2}$ of defects; see Eq. (40). The final result will provide us with a formula, Eq. (46), that expresses the pinning force density $F_{\text {pin }}$ due to defect pairs in terms of the individual pair forces $f_{\text {pair }}[g(\boldsymbol{\rho}), \boldsymbol{\Delta}]$ for defects separated by $\boldsymbol{\rho}$ and with a mismatch $\Delta$ between them and the vortices. While this expression can be evaluated precisely using numerical techniques, here we will discuss analytic results that are necessarily of approximate nature.

In the first step, we fix $\boldsymbol{\Delta}$ and average over the vector $\mathbf{x}=[x(X), b]$ in a similar fashion as in Sec. II B. For each $b$, we average the aggregated pinning force exerted by the two defects while pushing $X$ from large negative to large positive values and then take the average over the impact parameter $b$. This procedure provides us with the pinning force $\mathbf{f}_{\text {pair }}$ of a defect pair at fixed mismatch vector $\Delta$ and coupling $g$,

$$
\begin{aligned}
& \mathbf{f}_{\mathrm{pair}}(g, \boldsymbol{\Delta}) \\
& =\left\langle\mathbf{f}_{p}\left[\mathbf{x}+\frac{\boldsymbol{\Delta}}{2}+\mathbf{u}_{1}(\mathbf{x})\right]+\mathbf{f}_{p}\left[\mathbf{x}-\frac{\boldsymbol{\Delta}}{2}+\mathbf{u}_{2}(\mathbf{x})\right]\right\rangle_{\mathbf{x}},
\end{aligned}
$$

where $\mathbf{u}_{1}(\mathbf{x}), \mathbf{u}_{2}(\mathbf{x})$ are the solutions to the coupled system, Eq. (39). As before, this average involves the jumps in energy between bistable solutions for the pair-pinning problem defined by Eq. (39).

In step 2, we average over mismatch vectors $\boldsymbol{\Delta}$ (the normalization $a_{0}^{2}$ follows from $\boldsymbol{\Delta}$ being restricted to the unit cell of the vortex lattice),

$$
\left\langle f_{\text {pair }}(g, \boldsymbol{\Delta})\right\rangle_{\Delta}=\int \frac{d^{2} \Delta}{a_{0}^{2}} f_{\text {pair }}(g, \boldsymbol{\Delta}),
$$

where $f_{\text {pair }}=\mathbf{f}_{\text {pair }} \cdot\left(-\mathbf{e}_{x}\right)$ denotes the (negative) $x$ component of $\mathbf{f}_{\text {pair }}$; note that for a vortex lattice pushed along the positive $x$ direction, the $\mathbf{x}$-averaged pinning force points in the negative $x$ direction. The $y$ component of $\mathbf{f}_{\text {pair }}$ vanishes after the $\boldsymbol{\Delta}$ averaging since it is compensated by the configuration with $\boldsymbol{\Delta} \rightarrow-\boldsymbol{\Delta}$ and $b \rightarrow-b$.

Third, we determine the pinning force density $F_{\text {pin }}$ exerted by all defect pairs within a volume $V$ by summing over pairs that are pinned at different separation $\boldsymbol{\rho}=\left(\mathbf{R}_{\mu(1)}-\right.$ $\left.\mathbf{R}_{\mu(2)}, z_{1}-z_{2}\right)$, where $\mathbf{R}_{\mu(1)}-\mathbf{R}_{\mu(2)}$ refers to the separation between the vortices and $z_{1}-z_{2}$ is the distance between the defects along the $z$ axis. This final sum (or average) accounts for the dependence of the pair force $\mathbf{f}_{\text {pair }}(g, \boldsymbol{\Delta})$ on the coupling $g(\rho)$. Approximating the sum by an integral gives (note the factor of $\frac{1}{2}$ to avoid double counting of the defects)

$$
\begin{aligned}
F_{\text {pin }}= & \frac{n_{p}^{2}}{2 V} \int_{V} d^{2} \mathbf{R}_{\mu(1)} d^{2} \mathbf{R}_{\mu(2)} d z_{1} d z_{2} \\
& \times\left\langle f_{\text {pair }}\left[g\left(\mathbf{R}_{\mu(1)}-\mathbf{R}_{\mu(2)}, z_{1}-z_{2}\right), \boldsymbol{\Delta}\right]\right\rangle_{\Delta} .
\end{aligned}
$$

Carrying out one volume integral, we arrive at the final expression

$$
F_{\text {pin }}=\frac{n_{p}^{2}}{2} \int_{V} d^{3} \boldsymbol{\rho}\left\langle f_{\text {pair }}[g(\boldsymbol{\rho}), \boldsymbol{\Delta}]\right\rangle_{\boldsymbol{\Delta}} .
$$

It remains to solve the coupled equations (39) and determine the resulting pinning force $\mathbf{f}_{\text {pair }}(g, \boldsymbol{\Delta})$ of defect pairs that enters the final expression (46) for the pinning force density $F_{\text {pin }}$. We first provide a qualitative overview of the results, before presenting the detailed derivations.

\section{Overview of results}

Pinning is maximally strong if both defects are synchronized, i.e., $\boldsymbol{\Delta}=0$. In this case, $\mathbf{u}_{1}=\mathbf{u}_{2}=\mathbf{u}$ and Eq. (39) reduces to a single equation

$$
\bar{C} \mathbf{u}=(1+g) \mathbf{f}_{p}(\mathbf{x}+\mathbf{u}),
$$

which is equivalent to the single-defect problem with renormalized pinning strength

$$
\kappa_{\mathrm{eff}}(g, \boldsymbol{\Delta}=\mathbf{0})=\kappa(1+g) .
$$

The condition $\kappa_{\text {eff }}(g, \mathbf{0})>1$ for a strong pinning pair then requires $g(\boldsymbol{\rho})>g_{0}(\kappa)$ with

$$
g_{0}(\kappa)=\frac{1}{\kappa}-1
$$

restricting the maximal separation between the defects; see Fig. 6.

In order to arrive at an expression for $f_{\text {pair }}(g, \boldsymbol{\Delta}=\mathbf{0})$, it is convenient to express $\kappa_{\text {eff }}$ in terms of $g$ and the critical value $g_{0}$,

$$
\kappa_{\text {eff }}(g, \mathbf{0})=1+\frac{g-g_{0}(\kappa)}{1+g_{0}(\kappa)} .
$$

With $\kappa_{\text {eff }}$ above but close to unity, we can make use of Eq. (27) and find that

$$
\begin{aligned}
f_{\text {pair }}(g, \mathbf{0}) & \sim\left(\xi / a_{0}\right)^{2}\left[\kappa_{\text {eff }}(g, \mathbf{0})-1\right]^{2} f_{p} \\
& \sim\left(\xi / a_{0}\right)^{2}\left(g-g_{0}\right)^{2} f_{p}
\end{aligned}
$$

scales with $\left(g-g_{0}\right)^{2} \leqslant 1$.

Defect pairs satisfying $g(\boldsymbol{\rho})>g_{0}(\kappa)$ can pin strongly at a finite mismatch vector $\Delta$ as well. We then have to generalize the effective pair-pinning strength $\kappa_{\text {eff }}(g, \Delta)$ to finite $\Delta$ and the strong pinning condition $\kappa_{\text {eff }}(g, \boldsymbol{\Delta})=1$ will provide us with the $\boldsymbol{\Delta}(g)$ domain where pair pinning is strong. The latter will allow us to determine the pair-pinning force $f_{\text {pair }}(g, \boldsymbol{\Delta})$. However, a quantitative evaluation of $\kappa_{\text {eff }}(g, \boldsymbol{\Delta})$ for general $g$ and $\boldsymbol{\Delta}$ is quite cumbersome, given the complex geometry of the problem.

Progress can be made by carrying out a perturbative analysis in $\Delta \ll \xi$, which requires defect pairs to be at the verge of strong pinning, i.e., $g(\rho)-g_{0}(\kappa) \ll 1$. Such a calculation 


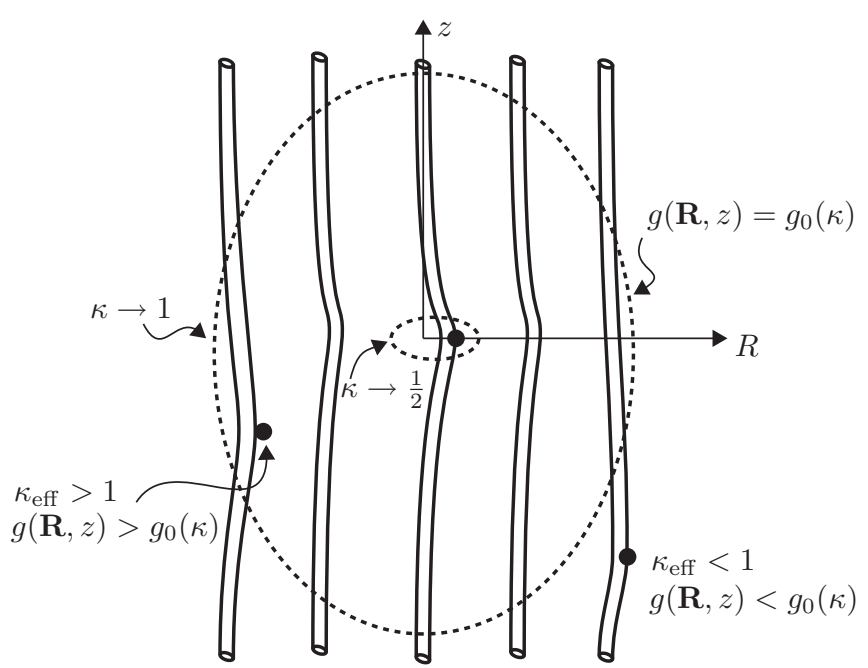

FIG. 6. Illustration of domains with different effective pairpinning strengths. A reference vortex with undisturbed transverse position $\mathbf{R}_{\mu}=\mathbf{0}$ is pinned by a defect at height $z=0$; see center of the figure. The surface $g(\mathbf{R}, z)=g_{0}(\kappa)$ (large ellipse) where the coupling is critical determines the boundary between strong and weak pinning by defect pairs. A defect located inside this region combines with the central defect to form a strong pinning pair with an effective Labusch parameter (or pinning strength) $\kappa_{\text {eff }}>1$, while a defect outside the region does not contribute to the strong pinning by rare events. For $\kappa \rightarrow 1$, the ellipse diverges to infinity as $R_{0}, z_{0} \propto(1-\kappa)^{-1}$; see Eq. (64). For $\kappa \rightarrow \frac{1}{2}$, the ellipse shrinks to $R \sim \xi$ and $z \sim\left(\kappa-\frac{1}{2}\right) a_{0}$.

for the effective pinning strength $\kappa_{\text {eff }}(g, \boldsymbol{\Delta})$ is carried out in Sec. III E; furthermore, it is shown there, that strong pinning with $\kappa_{\text {eff }}(g, \boldsymbol{\Delta})>1$ is limited to small mismatches $\Delta_{x}<\Delta_{x}^{0}$ and $\Delta_{y}<\Delta_{y}^{0}$ with [see Eqs. (98) and (103)]

$$
\begin{aligned}
& \Delta_{x}^{0} \sim \xi\left(g-g_{0}\right)^{1 / 2}\left(g+g_{0}\right), \\
& \Delta_{y}^{0} \sim \xi\left(g-g_{0}\right)^{1 / 2}\left(g+g_{0}\right)^{-1 / 2} ;
\end{aligned}
$$

see Fig. 7. An estimate for the $\Delta$-averaged pair force can be obtained by combining the maximal pair force (51) at $\boldsymbol{\Delta}=\mathbf{0}$

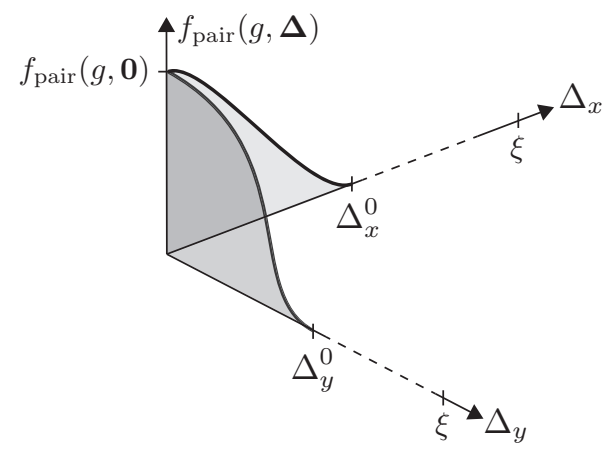

FIG. 7. Sketch of the pinning force $f_{\text {pair }}(g, \Delta)$ for a pair of defects on the verge of strong pair pinning, $g-g_{0} \ll 1$ as a function of the mismatch vector $\boldsymbol{\Delta}=\left(\Delta_{x}, \Delta_{y}\right)$. The pinning force for the pair-defect decays from its maximal value at $\boldsymbol{\Delta}=\mathbf{0}$, Eq. (51), on the scales $\Delta_{x}^{0}, \Delta_{y}^{0} \ll \xi$ given by Eqs. (52) that are small compared to the vortex core size $\xi$. and the region in $\Delta$ where pinning is strong (see Fig. 7),

$$
\begin{aligned}
\left\langle f_{\text {pair }}(g, \boldsymbol{\Delta})\right\rangle_{\Delta} & \sim \frac{\Delta_{x}^{0} \Delta_{y}^{0}}{a_{0}^{2}} f_{\text {pair }}(g, \mathbf{0}) \\
& \sim \frac{\xi^{4}}{a_{0}^{4}}\left(g-g_{0}\right)^{3}\left(g+g_{0}\right)^{1 / 2} f_{p} .
\end{aligned}
$$

A more precise result for the average pair-pinning force $\left\langle f_{\text {pair }}(g, \boldsymbol{\Delta})\right\rangle_{\boldsymbol{\Delta}}$ is derived in Sec. III F; see Eq. (111).

For $g(\rho)-g_{0}(\kappa) \sim O(1)$, the action of a defect-pair can be analyzed only on a qualitative level. Such large coupling $g$ implies that defects are nearby and the mismatch vector $\boldsymbol{\Delta}$ can go up to the vortex-core radius $\xi$. The averaging of the pair force $f_{\text {pair }} \sim\left(\xi / a_{0}\right)^{2} f_{p}$ [see Eq. (27)] for $\kappa-1$ of order unity over $\boldsymbol{\Delta}$ then gives

$$
\left\langle f_{\text {pair }}(g, \boldsymbol{\Delta})\right\rangle_{\boldsymbol{\Delta}} \sim\left(\xi / a_{0}\right)^{4} f_{p}
$$

Unlike in the previous case, a precise form for the dependencies of the effective pinning strength $\kappa_{\text {eff }}(g, \boldsymbol{\Delta})$ and the pair-pinning force $f_{\text {pair }}(g, \boldsymbol{\Delta})$ on the mismatch vector $\boldsymbol{\Delta}$ cannot be derived analytically. Quantitative insights can be made by a numerical treatment of the problem (see Sec. III F and Appendix B). Such proper averaging over the vector $\boldsymbol{\Delta}$ will provide only a numerical prefactor to the result in Eq. (54), which we do not consider here.

Remarkably, Eq. (53) as originally derived from a perturbative analysis in small $g(\rho)-g_{0}(\kappa) \ll 1$ produces the correct scaling result Eq. (54) also for $g(\rho)-g_{0}(\kappa) \sim O(1)$. On a qualitative level, we can thus extend the regime of applicability of the expression Eq. (53) to any coupling $g$ within the interval $\left[g_{0}, 1\right]$.

Substituting $\left\langle f_{\text {pair }}(g, \boldsymbol{\Delta})\right\rangle_{\boldsymbol{\Delta}}$ from (53) into Eq. (46) yields the pinning force density

$$
\begin{aligned}
F_{\text {pin }} & \sim\left(\frac{\xi}{a_{0}}\right)^{2}\left(n_{p} \xi^{2} a_{0}\right) n_{p} f_{p} \\
& \times \int_{g(\rho)>g_{0}} \frac{d^{3} \rho}{a_{0}^{3}}\left[g(\rho)-g_{0}\right]^{3}\left[g(\boldsymbol{\rho})+g_{0}\right]^{1 / 2},
\end{aligned}
$$

with the integration over distances $\rho$ restricted through the condition $g(\boldsymbol{\rho})>g_{0}$.

We now proceed to discuss the resulting pinning force density $F_{\text {pin }}$ due to the defect pairs. Of crucial importance in this discussion is the behavior of the coupling $g(\mathbf{R}, z)$ that is of order unity at distances $R, z<a_{0}$ and rapidly decays farther away; see Sec. II A. We distinguish three cases: (i) the limit $\kappa \rightarrow \frac{1}{2}$, where $g_{0} \rightarrow 1$, implying that the coupling $g>g_{0}$ has to be close to unity. This condition demands that relevant distances $R$ are far below $a_{0}$, from what follows that both defects pin the same vortex. This regime, where defect pairs act on the same vortex, extends throughout all of regime (ii), where $\frac{1}{2}<\kappa<1 /\left[1+g\left(R=a_{0}\right)\right]$; in this regime, the coupling $g$ is never large enough to produce strong pinning of different vortices that are always further apart than $a_{0}$. Finally, in region (iii), $\kappa \rightarrow 1$ and $g_{0} \rightarrow 0$, hence, even a small coupling $g$ is sufficient to establish a strong pinning defect pair. In this case, distances $R>a_{0}$ become relevant and different vortices can get strongly pinned to separated defects. 
Starting with region (i), we consider pinning strengths of individual defects close to the threshold $\kappa=\frac{1}{2}$. The expansion of Eq. (49) for $\kappa-\frac{1}{2} \ll 1$ gives the critical coupling $g_{0}(\kappa) \approx 1-4\left(\kappa-\frac{1}{2}\right)$ close to unity, and the condition $g(\rho)>$ $g_{0}(\kappa)$ requires both defects to act on the same vortex. Using Eq. (9) further implies that $g(\mathbf{0}, z)-g_{0} \approx 4\left(\kappa-\frac{1}{2}\right)-z / a_{h}>$ 0 , hence the maximal longitudinal separation is limited by $z_{0} \approx 4\left(\kappa-\frac{1}{2}\right) a_{h}$. Since for such range of coordinates we have $g(\rho)+g_{0} \sim O(1)$, the pinning force density in Eq. (55) can be cast into the form

$$
\begin{aligned}
F_{\text {pin }} & \sim\left(\frac{\xi}{a_{0}}\right)^{2}\left(n_{p} \xi^{2} a_{0}\right) n_{p} f_{p} \int_{0}^{z_{0}} \frac{d z}{a_{0}}\left[4\left(\kappa-\frac{1}{2}\right)-\frac{z}{a_{h}}\right]^{3} \\
& \sim\left(\frac{\xi}{a_{0}}\right)^{2}\left(\kappa-\frac{1}{2}\right)^{4}\left(n_{p} \xi^{2} a_{0}\right) n_{p} f_{p},
\end{aligned}
$$

where we have ignored the logarithmic factor in the expression for the healing length [see the definition of $a_{h}$ below Eq. (9)] and replaced $a_{h} \sim a_{0}$. The result in Eq. (56) defines the onset of the pinning force density due to defect pairs for $\kappa$ rising above $\kappa=\frac{1}{2}$; it starts dominating over the collective pinning result, Eq. (31), as soon as the pinning strength surpasses the threshold $\kappa=\frac{1}{2}$ by a small amount $\sim\left(a_{0} / \lambda\right)^{1 / 2}$.

Increasing $\kappa$ through region (ii), i.e., staying below the threshold $\kappa=1 /\left[1+g\left(R=a_{0}\right)\right]$, the critical coupling $g_{0}(\kappa)$ will remain finite, of order unity. Referring to Figs. 2 and 3 , we note that in this situation, the separation $\rho=(\mathbf{R}, z)$ between defects must remain within the peak region III, since otherwise $g$ rapidly decays to a value $\sim a_{0}^{2} / \lambda^{2} \ll 1$ and the criterion $g(\boldsymbol{\rho})>g_{0}(\kappa)$ cannot be met. The integral in Eq. (55) is of order unity and the pinning force density assumes the small pair or cluster value

$$
F_{\text {pin }}=F_{\text {clust }} \sim \frac{\xi^{2}}{a_{0}^{2}}\left(n_{p} \xi^{2} a_{0}\right) n_{p} f_{p} .
$$

Finally, when $\kappa$ resides within the small interval $1-$ $a_{0}^{2} / \lambda^{2} \lesssim \kappa \lesssim 1$, the critical coupling becomes small, $g_{0} \lesssim$ $a_{0}^{2} / \lambda^{2}$, and the separation between the defects producing strong pair-pinning extends beyond region III into regions I, II, and even the (yellow) nondispersive region of Fig. 2. In this case, which includes region (iii), we drop the small value $g_{0}(\kappa)$ against $g(\rho)$ in Eq. (55) and rewrite the result for the pinning force density as

$$
F_{\text {pin }} \sim\left(\frac{\xi}{a_{0}}\right)^{2}\left(n_{p} \xi^{2} a_{0}\right) n_{p} f_{p} \int_{g(\rho)>g_{0}} \frac{d^{2} \mathbf{R} d z}{a_{0}^{3}} g(\mathbf{R}, z)^{7 / 2} .
$$

The integration is dominated by small distances: the smallpair or cluster region (region III in Fig. 2) contributes the same estimate as in Eq. (57),

$$
F_{\text {III }} \sim F_{\text {clust }} .
$$

A similar contribution arises from region I: we integrate over the transverse coordinate $R<\sqrt{a_{0} z}$ and find the expression

$$
F_{\mathrm{I}} \sim F_{\text {clust }} \int_{a_{0}}^{\lambda^{2} / a_{0}} \frac{d z}{a_{0}} \frac{a_{0} z}{a_{0}^{2}}\left(\frac{a_{0}}{z}\right)^{7 / 2} \sim F_{\text {clust }},
$$

where the main contribution originates from the lower bound $z \sim a_{0}$ (and hence also $R \sim a_{0}$ ).
The contributions of region II and the nondispersive regime are smaller by a factor $\left(a_{0} / \lambda\right)^{3}$ : In region II with transverse and longitudinal extension $R \sim \lambda$ and $z \sim \lambda^{2} / a_{0}$, the Green's function assumes a constant value with $g^{7 / 2} \sim\left(a_{0} / \lambda\right)^{7}$ and the integration gives a result

$$
F_{\text {II }} \sim\left(\frac{a_{0}}{\lambda}\right)^{3} F_{\text {clust }} .
$$

In dealing with the nondispersive region, we introduce the rescaled distance $\tilde{\boldsymbol{\rho}}=\left[\mathbf{R},\left(a_{0} / 4 \sqrt{\pi} \lambda\right) z\right]$; the nondispersive region is bounded below by $|\tilde{\boldsymbol{\rho}}| \gtrsim \lambda$ and the condition $g(\tilde{\boldsymbol{\rho}})>$ $g_{0}(\kappa)$ translates to the upper boundary $|\tilde{\boldsymbol{\rho}}| \lesssim a_{0}^{2} / \lambda g_{0}$. The integral over the nondispersive region then takes the form

$$
\begin{aligned}
F_{\text {non-disp }} & \sim\left(\frac{\xi}{a_{0}}\right)^{2} n_{p}\left(n_{p} \xi^{2} a_{0}\right) f_{p} \int_{\tilde{\rho} \sim \lambda}^{\tilde{\rho} \sim a_{0}^{2} / \lambda g_{0}} \frac{d^{3} \tilde{\rho}}{a_{0}^{3}} \frac{\lambda}{a_{0}}\left(\frac{a_{0}^{2}}{\lambda \tilde{\rho}}\right)^{7 / 2} \\
& \sim\left(\frac{a_{0}}{\lambda}\right)^{3} F_{\text {clust }},
\end{aligned}
$$

which, given the large exponent $7 / 2$, is determined by the lower bound $\tilde{\boldsymbol{\rho}} \sim \lambda$. The total pinning force density then sums up to

$$
F_{\text {pin }} \sim F_{\text {I }}+F_{\text {III }} \sim F_{\text {clust }} .
$$

In the limit $\kappa \rightarrow 1$, we have $g_{0}(\kappa) \rightarrow 0$ and pairs of arbitrarily distant defects induce a finite pinning force. Indeed, the maximal distance between defects [providing the upper bound of the integral in Eq. (62)] diverges as $\tilde{\rho} \sim a_{0}^{2} / \lambda(1-\kappa)$; this translates into maximal longitudinal and transverse separations $z \leqslant z_{0}$ and $R \leqslant R_{0}$ between defects with

$$
z_{0} \sim \frac{a_{0}}{1-\kappa}, \quad R_{0} \sim \frac{a_{0}}{\lambda} \frac{a_{0}}{1-\kappa} ;
$$

see also Fig. 6. However, the pair-pinning force in Eq. (62) is dominated by the lower $\tilde{\rho}$ bound and contributions from distant defects are irrelevant, implying a finite integral even in the limit $\kappa \rightarrow 1$.

The origin of the power $\alpha=7 / 2$ can be traced back to the maximal size of the mismatch vector $\Delta$ ensuring strong pinning; see Eqs. (52). The derivation of the latter requires a detailed quantitative understanding of the pinning mechanism due to defect pairs at the verge of strong pinning that is presented in Secs. III D, III E, and III F below.

\section{Effective pinning potential $e_{\text {eff }}$}

In the following sections, we provide a systematic derivation of the results presented above. In a first step, we reduce the two-defect equation (39) to a single equation describing the interaction of a fictitious vortex with an effective pinning potential; see Fig. 5(b). We have seen that for vanishing mismatch $\boldsymbol{\Delta}$, the action of both defects is synchronized and the displacement of both vortices is identical, $\mathbf{u}_{1}=\mathbf{u}_{2}$. For a finite but small mismatch $\Delta$, we reformulate the problem in terms of the mean position $\mathbf{r}$ of the displaced vortices relative to the defects and the internal relative position $\delta \mathbf{r}$ (we remind that $\mathbf{x}_{i}=\mathbf{R}_{\mu(i)}-\mathbf{R}_{i}$ is the unperturbed defect - vortex distance),

$$
\begin{gathered}
\mathbf{r}=\frac{1}{2}\left(\mathbf{x}_{1}+\mathbf{u}_{1}+\mathbf{x}_{2}+\mathbf{u}_{2}\right), \\
\delta \mathbf{r}=\frac{1}{2}\left(\mathbf{x}_{1}+\mathbf{u}_{1}-\mathbf{x}_{2}-\mathbf{u}_{2}\right) .
\end{gathered}
$$


Solving perturbatively for the internal coordinate $\delta \mathbf{r}$ will allow us to reformulate the two-defect problem in terms of a single equation for the mean "fictitious" vortex tip position $\mathbf{r}$. This reformulated problem then will involve an effective pinning potential $e_{\text {eff }}(g, \boldsymbol{\Delta} ; \mathbf{r})$ exerting the pinning force $\mathbf{f}_{\mathrm{eff}}(\mathbf{r})=$ $-\nabla_{\mathbf{r}} e_{\text {eff }}(g, \boldsymbol{\Delta} ; \mathbf{r})$ on the "fictitious" vortex.

In the above new coordinates, the two-defect problem of Eqs. (39) takes the form

$$
\begin{gathered}
\bar{C}(\mathbf{r}+\delta \mathbf{r}-\mathbf{x}-\boldsymbol{\Delta} / 2)=\mathbf{f}_{p}(\mathbf{r}+\delta \mathbf{r})+g \mathbf{f}_{p}(\mathbf{r}-\delta \mathbf{r}), \\
\bar{C}(\mathbf{r}-\delta \mathbf{r}-\mathbf{x}+\boldsymbol{\Delta} / 2)=\mathbf{f}_{p}(\mathbf{r}-\delta \mathbf{r})+g \mathbf{f}_{p}(\mathbf{r}+\delta \mathbf{r}) .
\end{gathered}
$$

Expanding in $\delta \mathbf{r}$ to second order and subtracting one equation from the other provides us with an expression for $\delta \mathbf{r}$,

$$
\left[\delta_{i j}-\frac{1-g}{\bar{C}} \partial_{j} f_{p, i}(\mathbf{r})\right] \delta r_{j}=\frac{\Delta_{i}}{2}+O\left(\Delta^{3}\right) .
$$

We write the gradient of the radial pinning force $\mathbf{f}_{p}(r)=f_{p}(r) \hat{\mathbf{r}}$ (with $\hat{\mathbf{r}}=\mathbf{r} / r$ the unit vector in radial direction) in terms of the projectors $\mathcal{P}_{i j}^{\|}=\hat{r}_{i} \hat{r}_{j}$ and $\mathcal{P} \stackrel{\perp}{\perp}=\delta_{i j}-\hat{r}_{i} \hat{r}_{j}$,

$$
\partial_{j} f_{p, i}(\mathbf{r})=\mathcal{P}_{i j}^{\|} f_{p}^{\prime}(r)+\mathcal{P}_{i j}^{\perp} \frac{f_{p}(r)}{r} .
$$

Using $\delta_{i j}=\mathcal{P}_{i j}^{\|}+\mathcal{P}_{i j}^{\perp}$, Eq. (68) is rewritten as

$$
\left[\alpha_{\|}(r) \mathcal{P}_{i j}^{\|}+\alpha_{\perp}(r) \mathcal{P}_{i j}^{\perp}\right] \delta r_{j}=\frac{\Delta_{i}}{2}+O\left(\Delta^{3}\right)
$$

with

$$
\alpha_{\|}(r)=1-\frac{1-g}{\bar{C}} f_{p}^{\prime}(r), \quad \alpha_{\perp}(r)=1-\frac{1-g}{\bar{C}} \frac{f_{p}(r)}{r} .
$$

Making use of the relation $\mathcal{P}_{i j}^{\alpha} \mathcal{P}_{j k}^{\beta}=\delta_{i k} \delta_{\alpha \beta}$ for the projectors with $\alpha, \beta \in\{\|, \perp\}$ ), Eq. (70) is easily inverted and provides a relation between the internal coordinate $\delta \mathbf{r}$ and the mean coordinate $\mathbf{r}$ of the vortex pair,

$$
\delta \mathbf{r}=\left[\frac{\mathcal{P}^{\|}}{\alpha_{\|}(r)}+\frac{\mathcal{P}^{\perp}}{\alpha_{\perp}}\right] \frac{\Delta}{2}=\frac{(\hat{\mathbf{r}} \cdot \Delta) \hat{\mathbf{r}}}{2 \alpha_{\|}(r)}+\frac{\Delta-(\hat{\mathbf{r}} \cdot \Delta) \hat{\mathbf{r}}}{2 \alpha_{\perp}(r)} .
$$

Adding the two Eqs. (67) provides us with an equation for the mean vortex tip position,

$$
\bar{C}(\mathbf{r}-\mathbf{x})=\mathbf{f}_{\mathrm{eff}}(\mathbf{r}),
$$

reminiscent of the single-defect case but with an effective pinning force

$$
\mathbf{f}_{\mathrm{eff}}(\mathbf{r})=\frac{1}{2}(1+g)\left[\mathbf{f}_{p}(\mathbf{r}+\delta \mathbf{r})+\mathbf{f}_{p}(\mathbf{r}-\delta \mathbf{r})\right] .
$$

Expanding to second order in $\delta \mathbf{r}$ (or $\boldsymbol{\Delta}$ ), the effective pinning force becomes

$$
f_{\text {eff }, k}=(1+g) f_{p}(r) \hat{r}_{k}+\frac{1}{2}(1+g) \partial_{i} \partial_{j} f_{p, k}(\mathbf{r}) \delta r_{i} \delta r_{j},
$$

where the matrix of second derivatives of the pinning force can be expressed as $\partial_{i} \partial_{j} f_{p, k}(\mathbf{r})=\gamma \hat{r}_{i} \hat{r}_{j} \hat{r}_{k}+\mu\left(\hat{r}_{i} \delta_{j k}+\hat{r}_{j} \delta_{k i}+\right.$ $\left.\hat{r}_{k} \delta_{i j}\right)$ with

$$
\gamma=f_{p}^{\prime \prime}(r)-3 \partial_{r}\left[f_{p}(r) / r\right] \text { and } \mu=\partial_{r}\left[f_{p}(r) / r\right] .
$$

The sums $\partial_{i} \partial_{j} f_{p, k}(\mathbf{r}) \delta r_{i} \delta r_{j}$ involve the expressions

$$
\gamma \hat{r}_{i} \hat{r}_{j} \hat{r}_{k} \delta r_{i} \delta r_{j}=\gamma \hat{r}_{k} \frac{(\hat{\mathbf{r}} \cdot \boldsymbol{\Delta})^{2}}{4 \alpha_{\|}^{2}(r)},
$$

$$
\begin{aligned}
& \mu \delta_{i j} \hat{r}_{k} \delta r_{i} \delta r_{j}=\mu \hat{r}_{k}\left[\frac{(\hat{\mathbf{r}} \cdot \boldsymbol{\Delta})^{2}}{4 \alpha_{\|}^{2}(r)}+\frac{\boldsymbol{\Delta}^{2}-(\hat{\mathbf{r}} \cdot \boldsymbol{\Delta})^{2}}{4 \alpha_{\perp}(r)}\right], \\
& \mu\left(\delta_{j k} \hat{r}_{i}+\delta_{k i} \hat{r}_{j}\right) \delta r_{i} \delta r_{j} \\
& =\mu \frac{\hat{\mathbf{r}} \cdot \boldsymbol{\Delta}}{2 \alpha_{\|}(r)}\left[\frac{(\hat{\mathbf{r}} \cdot \boldsymbol{\Delta}) \hat{r}_{k}}{\alpha_{\|}(r)}+\frac{\Delta_{k}-(\hat{\mathbf{r}} \cdot \boldsymbol{\Delta}) \hat{r}_{k}}{2 \alpha_{\|}(r)}\right] .
\end{aligned}
$$

Combining Eqs. (75)-(79) gives the effective pinning force up to second order in the mismatch $\Delta$,

$$
\begin{aligned}
\mathbf{f}_{\mathrm{eff}}(\mathbf{r})= & (1+g)\left\{f_{p}(r) \hat{\mathbf{r}}+f_{p}^{\prime \prime}(r) \frac{(\hat{\mathbf{r}} \cdot \boldsymbol{\Delta})^{2}}{8 \alpha_{\|}^{2}(r)} \hat{\mathbf{r}}\right. \\
& +\partial_{r}\left[f_{p}(r) / r\right] \frac{\Delta^{2}-(\hat{\mathbf{r}} \cdot \boldsymbol{\Delta})^{2}}{8 \alpha_{\perp}^{2}(r)} \hat{\mathbf{r}} \\
& \left.+\partial_{r}\left[f_{p}(r) / r\right] \frac{\hat{\mathbf{r}} \cdot \boldsymbol{\Delta}}{4 \alpha_{\|}(r) \alpha_{\perp}(r)}[\boldsymbol{\Delta}-(\hat{\mathbf{r}} \cdot \boldsymbol{\Delta}) \hat{\mathbf{r}}]\right\},
\end{aligned}
$$

with the first three terms producing a radial force (along the vector $\hat{\mathbf{r}}$ ), while the last term contributes a transverse force [along the vector $\boldsymbol{\Delta}-(\hat{\mathbf{r}} \cdot \boldsymbol{\Delta}) \hat{\mathbf{r}}$ perpendicular to $\hat{\mathbf{r}}$ ].

This effective pinning force can be written as the gradient of the effective pinning potential $e_{\mathrm{eff}}(\mathbf{r}), \mathbf{f}_{\mathrm{eff}}(\mathbf{r})=-\nabla e_{\mathrm{eff}}(\mathbf{r})$, which has a much simpler form. Indeed, using $\nabla(\hat{\mathbf{r}} \cdot \boldsymbol{\Delta})=$ $[\boldsymbol{\Delta}-(\hat{\mathbf{r}} \cdot \boldsymbol{\Delta}) \hat{\mathbf{r}}] / r$ and fixing the integration constant by requiring $e_{\text {eff }}(\mathbf{r}) \rightarrow 0$ as $r \rightarrow \infty$, we find the effective pinning potential in the form

$$
\begin{aligned}
e_{\mathrm{eff}}(g, \boldsymbol{\Delta} ; \mathbf{r})= & (1+g) e_{p}(\mathbf{r})-\frac{\bar{C}(1+g)}{8(1-g)} \\
& \times\left[\frac{(\hat{\mathbf{r}} \cdot \boldsymbol{\Delta})^{2}}{\alpha_{\|}(r)}+\frac{\boldsymbol{\Delta}^{2}-(\hat{\mathbf{r}} \cdot \boldsymbol{\Delta})^{2}}{\alpha_{\perp}(r)}-\boldsymbol{\Delta}^{2}\right] .
\end{aligned}
$$

Using polar coordinates $\boldsymbol{\Delta}=\Delta(\cos \theta, \sin \theta)$ and $\mathbf{r}=$ $r(\cos \varphi, \sin \varphi)$, the effective potential can be described in terms of the magnitude $\Delta$ of the mismatch and the angle $\varphi-\theta$ enclosed by $\mathbf{r}$ and $\boldsymbol{\Delta}$,

$$
\begin{aligned}
e_{\mathrm{eff}}(g, \boldsymbol{\Delta} ; \mathbf{r}) & =(1+g) e_{p}(r)-\frac{\bar{C} \Delta^{2}(1+g)}{8(1-g)} \\
& \times\left[\frac{\cos ^{2}(\varphi-\theta)}{\alpha_{\|}(r)}+\frac{\sin ^{2}(\varphi-\theta)}{\alpha_{\perp}(r)}-1\right] .
\end{aligned}
$$

Inserting the expressions for $\alpha_{\|, \perp}$ [see Eq. (71)] and expanding for large distances $r \gg \xi$ [i.e., small values of $f_{p}^{\prime}(r)$, $\left.f_{p}(r) / r\right]$, we find that

$$
\begin{aligned}
e_{\mathrm{eff}}(g, \boldsymbol{\Delta} ; \mathbf{r}) \approx & (1+g) e_{p}(r)-(1+g) \frac{\Delta^{2}}{8} \\
& \times\left[f_{p}^{\prime}(r) \cos ^{2}(\varphi-\theta)+\frac{f_{p}(r)}{r} \sin ^{2}(\varphi-\theta)\right],
\end{aligned}
$$

with the anisotropic terms appearing at finite mismatch $\Delta$ proportional to $f_{p}^{\prime}(r), f_{p}(r) / r \propto(\xi / r)^{4}$ vanishing faster than the isotropic term $\propto e_{p}(r) \propto(\xi / r)^{2}$. 
In the limit $g=1$ (with defects acting on the same vortex at the same height $z$ ), the effective potential becomes

$$
\begin{aligned}
e_{\mathrm{eff}}(g=1, \boldsymbol{\Delta} ; \mathbf{r})= & 2 e_{p}(\mathbf{r})+\frac{1}{4}(\hat{\mathbf{r}} \cdot \boldsymbol{\Delta})^{2} f_{p}^{\prime}(r) \\
& +\frac{1}{4}\left[\boldsymbol{\Delta}^{2}-(\hat{\mathbf{r}} \cdot \boldsymbol{\Delta})^{2}\right] \frac{f_{p}(r)}{r},
\end{aligned}
$$

which corresponds, up to order $O\left(\boldsymbol{\Delta}^{2}\right)$, to the simple superposition of two mutually shifted pinning potentials, $e_{\text {eff }}(g=1, \boldsymbol{\Delta} ; \mathbf{r}) \approx e_{p}(\mathbf{r}+\boldsymbol{\Delta} / 2)+e_{p}(\mathbf{r}-\boldsymbol{\Delta} / 2)$. In the limit $g=0$, the two-defect problem (67) decouples and we can obtain independently each perturbed vortex tip position $\mathbf{r}_{i}=$ $\mathbf{x}_{i}+\mathbf{u}_{i}, i \in\{1,2\}$.

\section{E. Effective Labusch parameter $\kappa_{\text {eff }}$ and strong pinning range $\Delta^{0}$}

We proceed with the calculation of the effective Labusch parameter $\kappa_{\text {eff }}$ (or pinning strength) for the anisotropic pinning potential of Eq. (82). For a single isotropic defect, the Labusch parameter $\kappa$ is defined in Eq. (21) and involves the (maximal) potential curvature $f_{p}^{\prime}=-e_{p}^{\prime \prime}$ and the effective elasticity $\bar{C}$. Going to the defect pair, the anisotropic potential (82) depends on the distance and arrangement of defects through the parameters $g$ and $\boldsymbol{\Delta}=\Delta(\cos \theta, \sin \theta)$. In the following, we consider a vortex with an asymptotic trajectory $\mathbf{x}=(x, 0)$ and determine the angular dependence (on $\theta$ ) of the effective Labusch parameter $\kappa_{\text {eff }}(g, \boldsymbol{\Delta})=\kappa_{\text {eff }}(g, \Delta, \theta)$, i.e., we consider defect pairs with different angular arrangement relative to an asymptotically fixed vortex motion. Once the function $\kappa_{\text {eff }}(g, \boldsymbol{\Delta})$ is known, the condition $\kappa_{\text {eff }}\left(g, \Delta^{0}\right)=1$ will provide us with the maximal misfit $\boldsymbol{\Delta}^{0}(g)$ limiting the strong pinning range. Trajectories with different angle of incidence and/or finite impact parameter $b$ will be discussed later. Finally, the pinning strength in the vortex-defect system can be tuned by either changing the effective elasticity $\bar{C}$ or the energy scale $e_{p}$ of the defect potential-in the present discussion, we will tune $\kappa_{\text {eff }}$ via changing $\bar{C}$.

We start our derivation of the pinning strength $\kappa_{\text {eff }}(g, \Delta)$ by going back to its defining equation. For an isotropic pinning potential, this is given by (21), which in turn derives from the self-consistency equation $[4,5,28]$

$$
\bar{C}(r-x)=f_{p}(r) .
$$

Equation (85) allows us to connect incremental changes in the asymptotic and tip positions, $\delta x=\left[1-f_{p}^{\prime}(r) / \bar{C}\right] \delta r$, with jumps in $\delta r$ occurring when $1-f_{p}^{\prime}(r) / \bar{C}=0$. Combining this relation with the condition of its first appearance, $f_{p}^{\prime}(r) \rightarrow$ $\max \left[f^{\prime}(r)\right]$, leads to (21). Finally, the maximum force derivative $\max \left[f^{\prime}(r)\right]$ is achieved at the inflection point $r_{m}$ defined via $\left.f_{p}^{\prime \prime}\right|_{r_{m}}=0$.

In the present anisotropic situation, Eq. (85) has to be generalized to its vectorial form (73) and incremental changes in asymptotic and tip positions of vortices are related via

$$
\delta x_{i}=\left[\delta_{i j}+H_{i j}(\mathbf{r}) / \bar{C}\right] \delta r_{j},
$$

where

$$
H_{i j}(\mathbf{r})=\partial_{r_{i}} \partial_{r_{j}} e_{\text {eff }}(g, \Delta ; \mathbf{r})
$$

is the Hessian matrix associated with the pinning energy landscape $e_{\text {eff }}(g, \boldsymbol{\Delta} ; \mathbf{r})$. The Labusch criterion again marks the first appearance of an instability in the vortex tip position $\delta \mathbf{r}$. We thus have to invert Eq. (86) and find the solution $\delta \mathbf{r}(\delta \mathbf{x})-\mathrm{a}$ diverging result for $\delta \mathbf{r}$ then signals the presence of a jump in the vortex tip position. Approaching this divergence from the weak pinning domain, i.e., starting with a large $\bar{C}$ and decreasing its value, the jump appears when the determinant in the matrix relation (86) vanishes,

$$
\operatorname{det}\left[\bar{C} \delta_{i j}+H_{i j}(\mathbf{r})\right]=0 .
$$

Evaluating the Hessian in cylindrical coordinates $(r, \varphi)$, we obtain the matrix

$$
H=(1+g)\left[\begin{array}{cc}
-f_{p}^{\prime}+\beta \Delta^{2} & \gamma \Delta^{2} \\
\gamma \Delta^{2} & -f_{p}(r) / r+\delta \Delta^{2}
\end{array}\right],
$$

with

$$
\beta(r, \theta)=\frac{-1}{8}\left\{\left[\frac{f_{p}^{\prime \prime}(r)}{\alpha_{\|}^{2}(r)}\right]^{\prime} \cos ^{2} \theta+\left[\frac{\left(f_{p}(r) / r\right)^{\prime}}{\alpha_{\perp}^{2}(r)}\right]^{\prime} \sin ^{2} \theta\right\}
$$

and functions $\gamma(r, \theta), \delta(r, \theta)$ that we do not need to calculate explicitly. Above, we have made use of the fact that the tip trajectory stays always close to the $x$ axis (up to corrections of order $\left.\Delta^{2}\right)$, and, hence, we have set the angle $\varphi$ in $\beta(\mathbf{r}, \theta)$ to zero, $\beta(\mathbf{r}, \theta) \rightarrow \beta(r, \theta)$.

The condition of vanishing determinant (88) is equivalent to matching up the lower eigenvalue $\lambda_{-}(r, \theta)<0$ of $H$ with $\bar{C}$,

$$
\lambda_{-}(r, \theta)+\bar{C}=0
$$

furthermore, we need to find the location where this happens first, i.e., we have to determine the distance $r_{m}^{\text {eff }}(\theta)$ that generalizes $r_{m}$ to the anisotropic situation. Once this program is executed, the generalized Labusch parameter is given by

$$
\kappa_{\mathrm{eff}}(\theta)=\frac{-\lambda_{-}\left[r_{m}^{\mathrm{eff}}(\theta), \theta\right]}{\bar{C}},
$$

which assumes unity at the weak-to-strong pinning transition and larger values on decreasing $\bar{C}$ further into the strong pinning region.

Let us first consider the above generalized formulation of the Labusch parameter for the isotropic situation with $\Delta=$ 0 . Then $H_{i j}$ is already diagonal, with eigenvalues $\lambda_{-}(r)=$ $-f_{p}^{\prime}(r)<0$ and $\lambda_{+}(r)=-f_{p}(r) / r>0$ close to the inflection point $r_{m}$, where $f_{p}^{\prime \prime}\left(r_{m}\right)=0$ and the maximum in $-\lambda_{-}=f^{\prime}(r)$ is realized. These results are fully in line with the previous discussion of the Labusch criterion (21).

The perturbative analysis of the anisotropic situation contributes corrections to order $\Delta^{2}$ that introduce an angular dependence of the results on $\theta$. The eigenvalues $\lambda_{ \pm}(r, \theta)$ of $H$, see (89), coincide, to order $\Delta^{2}$, with its diagonal entries, since the off-diagonal terms only add a correction $\gamma^{2} \Delta^{4}$ to the determinant appearing in their calculation. In particular, the lower eigenvalue assumes the form

$$
\lambda_{-}(r, \theta) \approx(1+g)\left[-f_{p}^{\prime}(r)+\beta(r, \theta) \Delta^{2}\right] .
$$

Following the definition in Eq. (91), we have to evaluate this expression at the generalized inflection point $r_{m}^{\text {eff }}(\theta)$. The latter remains close to $r_{m}, r_{m}^{\text {eff }}(\theta)=r_{m}+O\left(\Delta^{2}\right)$, and using 
this Ansatz in (92), we find that the correction to $r_{m}$ is irrelevant since $f_{p}^{\prime}\left[r_{m}^{\text {eff }}(\theta)\right]=f_{p}^{\prime}\left[r_{m}+O\left(\Delta^{2}\right)\right] \approx f_{p}^{\prime}\left(r_{m}\right)+O\left(\Delta^{4}\right)$ as $f_{p}^{\prime \prime}\left(r_{m}\right)=0$. We thus arrive at the formal expression for the effective Labusch parameter

$$
\kappa_{\mathrm{eff}}(g, \boldsymbol{\Delta}) \approx \frac{1+g}{\bar{C}}\left[f_{p}^{\prime}\left(r_{m}\right)-\beta\left(r_{m}, \theta\right) \Delta^{2}\right] .
$$

Inserting the expression for $\beta(r, \theta)$ from above, we can rewrite this result into the convenient form

$$
\left.\kappa_{\mathrm{eff}}(g, \boldsymbol{\Delta}) \approx \frac{-\partial_{r}^{2} e_{\mathrm{eff}}(g, \boldsymbol{\Delta} ; r)}{\bar{C}}\right|_{r_{m}} .
$$

When the vortex trajectory is oriented at a finite angle $\phi$ with respect to the $x$ axis, the angular dependence in (94) has to be replaced according to $\theta \rightarrow \theta-\phi$. Finally, the discussion for a finite impact parameter $b$ can be easily reduced to the situation where the vortex approaches the defect center from an angle; see the discussion in Sec. III F below.

Note that, while $r_{m}^{\text {eff }} \approx r_{m}$ does not depend on angle to order $\Delta^{2}$, the effective pinning strength $\kappa_{\text {eff }}(g, \Delta, \theta-\phi)$ experienced by a vortex incident at an angle $\phi$ does. This implies that pinning is not equally strong when approaching the same defect from different directions. Rather, $\kappa_{\text {eff }}$ may be larger (or smaller) than unity when changing $\phi \in[0,2 \pi]$. As a result, vortex trajectories crossing the same defect may undergo pinning and depinning jumps in some directions but not in other. In this context, we draw attention to another variant of a weak to strong pinning crossover as discussed $[38,39]$ in the context of random landscapes (rather than individual pinning defects or defect pairs), where Eq. (90) shows up in the discussion of zero modes triggering avalanches in the motion of a driven elastic line pinned in a random 2D potential landscape.

Next, we return to our vortex incident along $x$, substitute the anisotropic defect-pair potential Eq. (82) into the expression (94), and use $\kappa=f_{p}^{\prime}\left(r_{m}\right) / \bar{C}$ to find the explicit result

$$
\begin{aligned}
\kappa_{\mathrm{eff}}(g, \Delta, \theta) & \approx(1+g) \kappa+(1+g) \frac{\Delta^{2}}{8 \bar{C}} \\
& \times\left\{\frac{f_{p}^{\prime \prime \prime}}{\alpha_{\|}^{2}} \cos ^{2} \theta+\partial_{r}\left[\frac{\partial_{r}\left(f_{p} / r\right)}{\alpha_{\perp}^{2}}\right] \sin ^{2} \theta\right\}_{r=r_{m}} .
\end{aligned}
$$

Setting the pinning strength to its critical value, $\kappa_{\mathrm{eff}}(g, \boldsymbol{\Delta})=$ 1 , we now can determine the misfit parameter $\boldsymbol{\Delta}^{0}(g)$ below which pinning is strong. We first analyze the two special cases $\theta=0$ and $\theta=\pi / 2$, where the mismatch $\boldsymbol{\Delta}$ is parallel and perpendicular to the vortex trajectory, before generalizing the result to other angles $\theta$.

For $\theta=0$, we obtain

$$
\kappa_{\mathrm{eff}}(g, \Delta, 0) \approx(1+g) \kappa+(1+g) \frac{\Delta^{2}}{8 \bar{C}} \frac{f_{p}^{\prime \prime \prime}\left(r_{m}\right)}{[1-\kappa(1-g)]^{2}} .
$$

Since $f_{p}^{\prime \prime \prime}\left(r_{m}\right)<0$, the pinning strength decreases below its maximal value $(1+g) \kappa$ as the mismatch $\Delta$ is increased. By setting $\kappa_{\text {eff }}(g, \Delta, 0)=1$ in Eq. (96), we can obtain the maximum longitudinal mismatch $\Delta_{x}^{0}$ below which the pinning by the two defects is strong,

$$
\Delta_{x}^{0}=\frac{\left(8 \bar{C} \kappa^{3}\right)^{1 / 2}}{\left[-f_{p}^{\prime \prime \prime}\left(r_{m}\right)\right]^{1 / 2}} \frac{\left(g-g_{0}\right)^{1 / 2}\left(g+g_{0}\right)}{(1+g)^{1 / 2}} .
$$

Estimating $f_{p}^{\prime \prime \prime} \sim f_{p} / \xi^{3}$ and $f_{p} / \bar{C} \xi \sim O(1)$ and dropping the factor $1+g$ in the denominator, we can express the relevant $g$ dependence of $\Delta_{x}^{0}$ in the parametric form

$$
\Delta_{x}^{0} \sim \xi\left(g-g_{0}\right)^{1 / 2}\left(g+g_{0}\right) .
$$

For $\theta=\pi / 2$, we first rewrite the prefactor of the $\sin ^{2} \theta$ term in Eq. (95). Introducing the notation $\beta(r)=f_{p}(r) / r$, we find that

$$
\begin{aligned}
& \partial_{r}\left(\alpha_{\perp}^{-2} \beta^{\prime}\right)=\alpha_{\perp}^{-3}\left(\alpha_{\perp} \beta^{\prime \prime}-2 \alpha_{\perp}^{\prime} \beta^{\prime}\right) \\
& =\alpha_{\perp}^{-3} \bar{C}^{-1}\left[\bar{C} \beta^{\prime \prime}-(1-g) \beta \beta^{\prime \prime}+2(1-g)\left(\beta^{\prime}\right)^{2}\right] .
\end{aligned}
$$

Expressing the effective elasticity $\bar{C}$ through $\kappa$ and $\beta, \bar{C}=$ $\kappa^{-1}(\beta r)_{r=r_{m}}^{\prime}$, the second factor in this expression simplifies to

$$
\begin{aligned}
& \kappa^{-1}(\beta r)^{\prime} \beta^{\prime \prime}-(1-g) \beta \beta^{\prime \prime}+2(1-g)\left(\beta^{\prime}\right)^{2} \\
& =\left(\kappa^{-1}+g-1\right)(\beta r)^{\prime} \beta^{\prime \prime}+(1-g)\left[2\left(\beta^{\prime}\right)^{2}+(\beta r)^{\prime} \beta^{\prime \prime}-\beta \beta^{\prime \prime}\right] \\
& =\left(g+g_{0}\right)(\beta r)^{\prime} \beta^{\prime \prime}+(1-g) \beta^{\prime}(r \beta)^{\prime \prime} \\
& =\left(g+g_{0}\right)(\beta r)^{\prime} \beta^{\prime \prime}=-\left(2 \kappa \bar{C} / r_{m}\right)\left(g+g_{0}\right) \beta^{\prime},
\end{aligned}
$$

where in the last two steps we used $(r \beta)^{\prime \prime}=f_{p}^{\prime \prime}\left(r_{m}\right)=0$ and $\beta^{\prime \prime}=-(2 / r) \beta^{\prime}$. Since $f_{p}^{\prime}\left(r_{m}\right)>0$, we have $\beta^{\prime}>0$ at $r=r_{m}$, and hence the effective Labusch parameter

$$
\begin{aligned}
\kappa_{\text {eff }}(g, \Delta, \pi / 2) & =(1+g) \kappa \\
& -(1+g) \frac{\Delta^{2}}{4 \bar{C}} \frac{\kappa\left(g+g_{0}\right) \beta^{\prime}\left(r_{m}\right) / r_{m}}{\left[1-\beta\left(r_{m}\right)(1-g) / C\right]^{3}}
\end{aligned}
$$

again decreases with increasing $\Delta$. Setting $\kappa_{\mathrm{eff}}(\pi / 2)=1$ then defines the maximal transverse mismatch for strong pinning,

$$
\Delta_{y}^{0}=\frac{2 \bar{C}^{1 / 2}\left[1-\beta\left(r_{m}\right)(1-g) / \bar{C}\right]^{3 / 2}}{\left[\beta^{\prime}\left(r_{m}\right) / r_{m}\right]^{1 / 2}} \frac{\left(g-g_{0}\right)^{1 / 2}}{\left[(1+g)\left(g+g_{0}\right)\right]^{1 / 2}} .
$$

Using similar estimates as above, we obtain the result in parametric form

$$
\Delta_{y}^{0} \sim \xi \frac{\left(g-g_{0}\right)^{1 / 2}}{\left(g+g_{0}\right)^{1 / 2}} .
$$

Finally, expressing the effective Labusch parameter in Eq. (95) in terms of the maximum longitudinal and transverse mismatches $\Delta_{x}^{0}, \Delta_{y}^{0}$ in Eqs. (97) and (102) yields the angular dependence

$$
\kappa_{\mathrm{eff}}(\theta)=1+\kappa\left(g-g_{0}\right)\left[1-\frac{\Delta^{2} \cos ^{2} \theta}{\left(\Delta_{x}^{0}\right)^{2}}-\frac{\Delta^{2} \sin ^{2} \theta}{\left(\Delta_{y}^{0}\right)^{2}}\right] .
$$

It is interesting to compare the pinning strengths for different vortex - defect configurations where the vortex trajectories are either parallel or perpendicular to the mismatch vector $\boldsymbol{\Delta}$. Combining Eqs. (97) and (102) and using $g_{0}=1 / \kappa-1$, we obtain the following ratio for the maximal longitudinal and transverse mismatches:

$$
\frac{\Delta_{y}^{0}}{\Delta_{x}^{0}}=\frac{1}{\sqrt{2}} \frac{\left[-f_{p}^{\prime \prime \prime}\left(r_{m}\right)\right]^{1 / 2}}{\left[\beta^{\prime}\left(r_{m}\right) / r_{m}\right]^{1 / 2}}\left[\frac{1-\beta\left(r_{m}\right)(1-g) / \bar{C}}{1-\kappa(1-g)}\right]^{3 / 2} .
$$




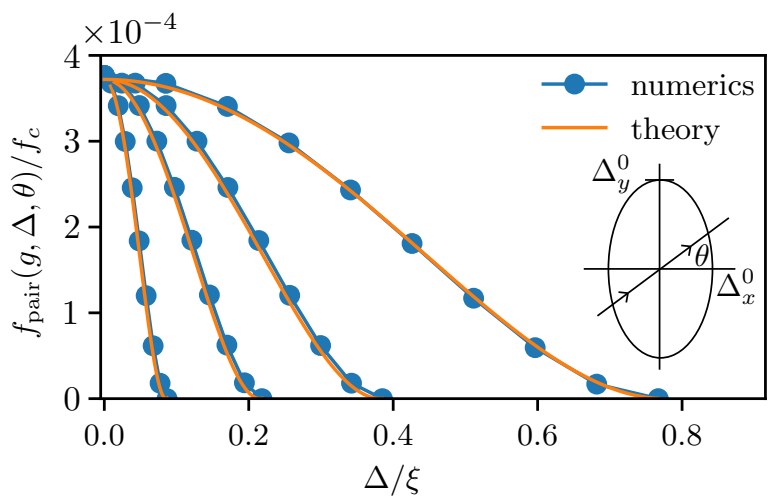

FIG. 8. Comparison of numerical (see Appendix B) and analytical [see Eq. (108)] results of the effective pinning force for a "fictitious" vortex trajectory $\mathbf{x}=[x(X), 0]$ passing through the center of the effective pinning potential. Results are shown for $\kappa=0.8$ and a small coupling $g=g_{0}(\kappa)+2 \times 10^{-2}$, producing a $\kappa_{\text {eff }}(g, \mathbf{0})$ [Eq. (50)] slightly above unity. In this regime, the maximal longitudinal and transverse mismatches are related by $\Delta_{x}^{0} \ll \Delta_{y}^{0}$. The mismatch vector $\boldsymbol{\Delta}=(\Delta \cos \theta, \Delta \sin \theta)$ encloses an angle $\theta$ with the $x$ direction: the four curves correspond to (from left to right; see also the inset) $\theta=0,3 \pi / 8,7 \pi / 16$, and $\pi / 2$. The pinning force is normalized by the scale $f_{c}=\left(\xi / a_{0}\right) f_{p}$.

For a maximal coupling between defects, $g=1$, such that the defect potentials directly add up [cf. Eq. (84)], the factor $[\cdots]^{3 / 2}$ in the above expression is unity. The ratio of the two scales then depends on the specific form of the pinning potential in the vicinity of the inflection point $r_{m}$. For the Lorentzian pinning potential producing the pinning force in Eq. (16), the ratio of the two scales reads $\Delta_{y}^{0} /\left.\Delta_{x}^{0}\right|_{g=1}=\sqrt{3 / 2}$ and pinning is always stronger, i.e., $\kappa_{\text {eff }}(\theta)$ is larger along the direction perpendicular to the mismatch vector. Furthermore, since $\beta\left(r_{m}\right)<0$, the ratio $\Delta_{y}^{0} / \Delta_{x}^{0}$ grows as $g$ is decreased away from unity towards its minimum value $g_{0}=1 / \kappa-1$. When $g=g_{0}$, the term $1-\kappa(1-g)$ in the denominator of Eq. (105) takes the value $2(1-\kappa)$, hence as $\kappa \rightarrow 1$, pinning due to pairs of distant defects is always stronger in the direction perpendicular to the mismatch, regardless of the specific form of the pinning potential.

The maximal longitudinal and transverse mismatches $\Delta_{x}^{0}$ and $\Delta_{y}^{0}$ allow us to identify the region of applicability of the perturbative approach that we have used to derive the effective pinning potential. For a longitudinal mismatch with $\theta=0$, Eq. (72) tells us that $\delta r \leqslant \Delta_{x}^{0} / 2 \alpha_{\|}$, and using the bound $\alpha_{\|} \geqslant 1-\kappa(1-g)=\kappa\left(g+g_{0}\right)$, we find that $\delta r \lesssim$ $\xi\left(g-g_{0}\right)^{1 / 2}$. The perturbative approach is valid provided that $\delta r \ll \xi$, which is the case for $g-g_{0} \ll 1$. Similarly, for a transverse mismatch, we use $\alpha_{\perp} \sim O(1)$ and therefore $\delta r \leqslant$ $\Delta_{y}^{0} / 2 \alpha_{\perp} \sim \Delta_{y}^{0}$. The perturbation $\delta r$ then again remains small for $g-g_{0} \ll 1$, except for the crossover to strong pinning where $\kappa=1, g_{0}=0$, and $\Delta_{y}^{0} \sim \xi$. In this case, the validity of the perturbative approach requires $\Delta_{y} \ll \xi$ [see Eq. (72)]; however, a comparison with the numerical results in Fig. 8 demonstrates that the perturbative approach still provides excellent agreement even for a mismatch $\Delta_{y}$ comparable with $\xi$.

\section{F. Average pinning force}

In this section, we use the findings on the effective Labusch parameter to calculate the average pinning force due to two defects coupled by $g$. Restricting first to vortex trajectories $\mathbf{x}=[x(X), 0]$ passing through the center of the effective pinning potential, we evaluate the $x$-averaged pinning force for the longitudinal component (along the $x$ direction) [see Eq. (22)],

$$
\begin{aligned}
f_{\text {pair }}(g, \Delta, \theta, b=0) \\
=\left(-\mathbf{e}_{x}\right) \cdot\left\langle\mathbf{f}_{p}[\mathbf{r}(x)+\delta \mathbf{r}(x)]+\mathbf{f}_{p}[\mathbf{r}(x)-\delta \mathbf{r}(x)]\right\rangle_{x} \\
=\frac{2}{1+g}\left\langle-f_{\mathrm{eff}, x}[\mathbf{r}(x)]\right\rangle_{x} \\
=\frac{2}{1+g} \frac{\Delta e_{\text {pin }}^{\mathrm{eff}, 1}+\Delta e_{\mathrm{pin}}^{\mathrm{eff}, 2}}{a_{0}},
\end{aligned}
$$

where $\Delta e_{\mathrm{pin}}^{\mathrm{eff}, 1}$ and $\Delta e_{\mathrm{pin}}^{\mathrm{eff}, 2}$ denote the jumps in the effective pinning potential defined as in Eq. (19), i.e., $e_{\text {pin }}^{\text {eff }}(\mathbf{x})=$ $e_{\text {eff }}[\mathbf{r}(\mathbf{x})]+\frac{1}{2} \bar{C}[\mathbf{x}-\mathbf{r}(x)]^{2}$.

Provided that $\kappa_{\mathrm{eff}}(g, \Delta, \theta)>1$, the jumps $\Delta e_{\mathrm{pin}}^{\mathrm{eff}, i}, i \in\{1,2\}$ in the pinning energy are given by Eq. (26) with the replacements $f_{p}^{\prime \prime \prime}\left(r_{m}\right) \rightarrow f_{\text {eff }}^{\prime \prime \prime}\left(r_{m}\right)$ and $\kappa \rightarrow \kappa_{\text {eff }}$; otherwise, for $\kappa_{\text {eff }}(g, \Delta, \theta)<1$, the effective pinning force vanishes after the averaging. This gives the two-defect pinning force

$$
\begin{aligned}
f_{\text {pair }}(g, \Delta, \theta, 0) & =\frac{18 \bar{C}^{2}}{(1+g) a_{0}\left[-f_{\text {eff }}^{\prime \prime \prime}\left(r_{m}\right)\right]} \\
& \times\left\{\max \left[0, \kappa_{\text {eff }}(\theta)-1\right]\right\}^{2} .
\end{aligned}
$$

Using Eq. (104) for the effective Labusch parameter and noting that $f_{\text {eff }}^{\prime \prime \prime}\left(r_{m}\right)=(1+g) f_{p}^{\prime \prime \prime}\left(r_{m}\right)+O\left(\Delta^{2}\right)$, we obtain

$$
\begin{aligned}
f_{\text {pair }}(g, \Delta, \theta, 0) \approx & \frac{18(\kappa \bar{C})^{2}}{(1+g)^{2} a_{0}\left[-f_{p}^{\prime \prime \prime}\left(r_{m}\right)\right]}\left(g-g_{0}\right)^{2} \\
& \times\left\{\max \left[0,1-\frac{\Delta^{2} \cos ^{2} \theta}{\left(\Delta_{x}^{0}\right)^{2}}-\frac{\Delta^{2} \sin ^{2} \theta}{\left(\Delta_{y}^{0}\right)^{2}}\right]\right\}^{2} .
\end{aligned}
$$

The pinning force thus decays with $\Delta$ from its maximal value $\sim f_{p}\left(g-g_{0}\right)^{2}$ at $\boldsymbol{\Delta}=0$ to zero as the mismatch increases to $\Delta_{x}^{0}$ and $\Delta_{y}^{0}$ along and perpendicular to the vortex trajectory, respectively; see Fig. 7. In Fig. 8, we compare this analytic formula to the numerical results (see Sec. B) in the regime $g-g_{0} \ll 1$ for different angles $\theta$. Note that, while the (perturbative) analytic results assume a small mismatch $\Delta \ll \xi$, they remain applicable for angles close to $\theta=\pi / 2$, where $\Delta$ becomes comparable to $\xi$.

The geometric complexity arising at finite impact $b$ (see Fig. 9) produces interesting new features, e.g., asymmetric pinning and depinning jumps or even trajectories with only one of the pinning/depinning jumps realized. In the generic situation, the vortex tip associated with the trajectory $\mathbf{x}=$ $[x(X), b]$ undergoes a jump every time the position $\mathbf{r}$ hits the distance $r_{m}+O\left(\Delta^{2}\right)$ from the center of the effective pinning potential. At the instance of the jump, the vortex asymptotic position is $\mathbf{x}=\mathbf{r}-\mathbf{f}_{\text {eff }}(\mathbf{r}) / \bar{C}$ [see Eq. (73)], i.e., at a distance $x_{p}=r_{m}-(1+g) f_{p}\left(r_{m}\right) / \bar{C}+O\left(\boldsymbol{\Delta}^{2}\right)$ from the 


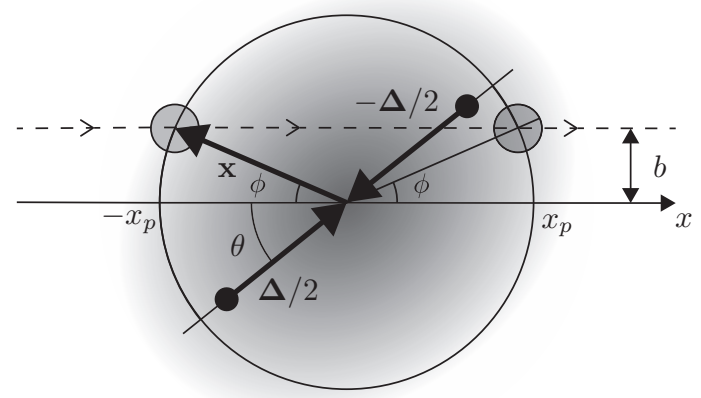

FIG. 9. Pinning and depinning of a "fictitious" vortex moving along the trajectory $\mathbf{x}=(x, b)$ with a nonvanishing impact parameter $b>0$ across a defect characterized by an effective pinning potential $e_{\text {eff }}(g, \boldsymbol{\Delta}, \mathbf{r})$ (shaded grey). The vortex is pinned and depinned from the defect at the intersection points of the trajectory with the circle (up to corrections of order $\Delta^{2}$ ) of radius $x_{p}$. The angular distance of the vortex from the mismatch vector $\Delta=(\Delta \cos \theta, \Delta \sin \theta)$ at these points is $\theta+\phi$ and $\theta-\phi$, with $\phi=\arcsin \left(b / x_{p}\right)$.

center of the pinning potential. Pinning and depinning then occur at the asymptotic positions $\mathbf{x}=\left( \pm x_{p} \cos \phi, x_{p} \sin \phi\right)$, with $\phi \approx \arcsin \left(b / x_{p}\right)$. Note that for the single-defect pinning in Fig. 4 corresponding to a large $\kappa$, the energy jumps associated with pinning and depinning appear at different asymptotic distances $x_{-}, x_{+}$from the defect center; for marginally strong effective pinning with $\kappa_{\text {eff }}(g, \Delta)-1 \ll 1$, we can neglect the difference $x_{+}-x_{-} \sim \xi\left(\kappa_{\text {eff }}-1\right)^{3 / 2}$ (see Refs. [27,33]) and set $x_{-} \approx x_{+} \approx x_{p}$.

The angles enclosed between $\mathbf{x}$ and $\boldsymbol{\Delta}$ at the pinning and depinning events are $\theta_{p}=\theta+\phi$ and $\theta_{\mathrm{dp}}=\theta-\phi$; see Fig. 9. The jump size at the pinning transition does not depend on the direction of the vortex motion and thus can be evaluated from the vortex trajectory passing directly through the center of the effective pinning potential but at an angle $\theta+\phi$, i.e., $\Delta e_{\mathrm{eff}}^{1}(g, \Delta, \theta, b)=\Delta e_{\mathrm{eff}}^{1}(g, \Delta, \theta+$ $\phi, 0)$. Similarly, $\Delta e_{\text {eff }}^{2}(g, \Delta, \theta, b)=\Delta e_{\text {eff }}^{2}(g, \Delta, \theta-\phi, 0)$ at depinning. Hence, the pinning and depinning jumps assume different values at finite impact $b$. The pinning force is then expressed as $f_{\text {pair }}(g, \Delta, \theta, b)=\frac{1}{2}\left[\Delta e_{\text {eff }}^{1}(g, \Delta, \theta+\right.$ $\left.\phi, 0)+\Delta e_{\mathrm{eff}}^{2}(g, \Delta, \theta-\phi, 0)\right] / a_{0}$. Furthermore, since for marginally strong pinning and $b=0$ trajectories, the pinning and depinning jumps in energy are equally sized, we express the resulting pinning force in terms of the forces exerted on the $b=0$ trajectories,

$$
\begin{aligned}
f_{\text {pair }}(g, \Delta, \theta, b)= & \frac{1}{2}\left[f_{\text {pair }}(g, \Delta, \theta+\phi, 0)\right. \\
& \left.+f_{\text {pair }}(g, \Delta, \theta-\phi, 0)\right] .
\end{aligned}
$$

With the pinning and depinning jumps no longer equal, we may encounter situations where one of the jumps is absent. This is the case for misfits $\Delta$ with $\theta \neq 0$ and $b \neq 0$; e.g., when $\kappa_{\text {eff }}(g, \Delta, \theta+\phi)>1$ but $\kappa_{\text {eff }}(g, \Delta, \theta-\phi)<1$, the vortex undergoes a pinning jump in energy when its asymptotic trajectory passes the circle of radius $x_{p}$ for the first time but does not undergo any depinning jump when the asymptotic trajectory crosses the circle a second time; see Fig. 10(b) (note that the corresponding trajectory with opposite impact
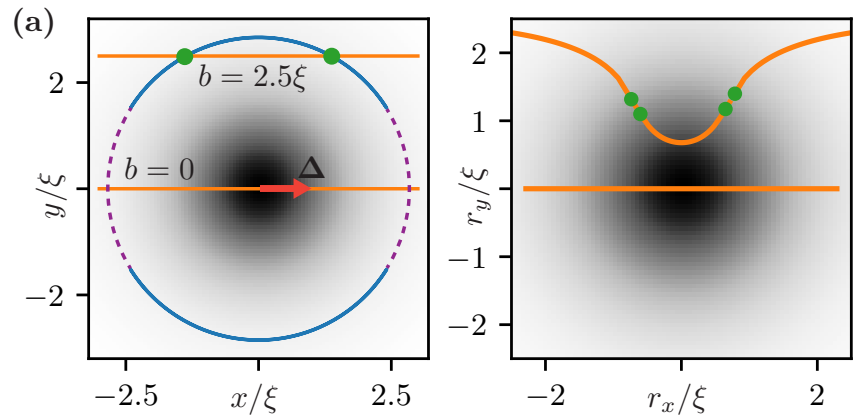

(b)
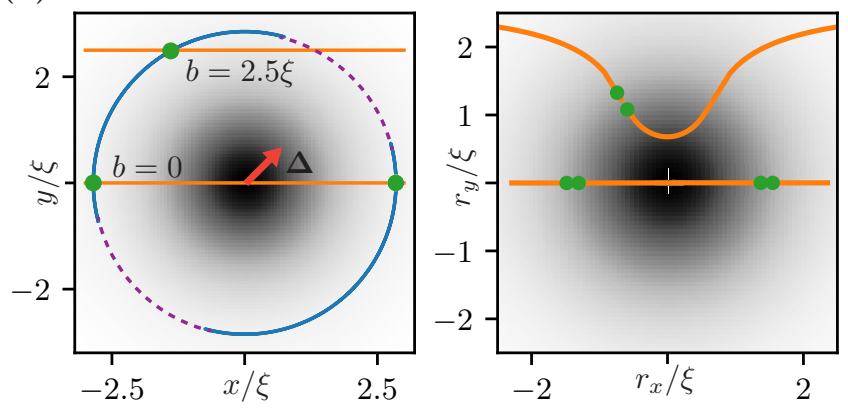

FIG. 10. Examples of vortex trajectories for an effective pinning potential due to two weak defects with $\kappa=0.8, g=g_{0}(\kappa)+2.10^{-2}$, $\Delta=0.1 \xi$. (a) Longitudinal mismatch vector $\Delta=(\Delta, 0)$ (red arrow, not to scale). For this setting, $\kappa_{\text {eff }}(g, \Delta, 0)=0.994$ at $b=0$ and the vortex does not undergo any jumps in energy when the asymptotic position crosses the circle of radius $x_{p}$ (dashed purple line). The plot on the right shows the vortex tip position $\mathbf{r}$ which remains continuous. A vortex passing at the transverse distance $b=2.5 \xi$ experiences an effective Labusch parameter $\kappa_{\text {eff }}(g, \Delta, \phi)=1.011$ [with $\left.\phi=\arcsin \left(b / x_{p}\right)\right]$, and the vortex undergoes pinning and depinning jumps (green dots) when its asymptotic position crosses the circle of radius $x_{p}$. The blue arcs denote the sections on the circle of radius $x_{p}$ where the effective pinning is strong; for $g-g_{0} \ll 1$ and $\kappa$ approaching unity, pinning is stronger along the direction perpendicular to the mismatch vector $\Delta$ (i.e., for $\phi=\pi / 2$ ) than along the direction parallel to the mismatch; see the discussion below Eq. (104). The tip position (right plot) jumps at the pinning and depinning events, as illustrated by the two pairs of green dots. (b) Mismatch vector enclosing an angle $\theta=\pi / 4$ with the vortex trajectory. At this angle, pinning is strong, $\kappa_{\mathrm{eff}}(g, \Delta, \theta)=1.005$, and a vortex with vanishing impact parameter $b=0$ undergoes both pinning and depinning jumps. However, for the vortex passing at $b=2.5 \xi$, the corresponding effective Labusch parameters read $\kappa_{\text {eff }}(g, \Delta, \theta+\phi)=1.014$ and $\kappa_{\text {eff }}(g, \Delta, \theta-\phi)=0.996$ and the vortex undergoes a jump only upon pinning. The right plot shows the corresponding tip trajectories with its jumps.

parameter $y=-b$ will undergo a depinning jump but will not jump upon pinning).

The pinning force averaging is done through integration over the mismatch $\Delta$ and the impact parameter $b$,

$$
\begin{aligned}
& \left\langle f_{\text {pair }}(g, \boldsymbol{\Delta})\right\rangle_{\Delta} \\
= & \int_{-x_{p}}^{x_{p}} \frac{d b}{a_{0}} \int_{-\pi}^{\pi} d \theta \int \frac{\Delta d \Delta}{a_{0}^{2}} f_{\text {pair }}(g, \Delta, \theta, b)
\end{aligned}
$$




$$
\begin{aligned}
= & \frac{x_{p}}{2 a_{0}} \int_{-\pi / 2}^{\pi / 2} d \sin \phi \int_{-\pi}^{\pi} d \theta \int \frac{\Delta d \Delta}{a_{0}^{2}}\left[f_{\text {pair }}(g, \Delta, \theta+\phi, 0)\right. \\
& \left.+f_{\text {pair }}(g, \Delta, \theta-\phi, 0)\right] \\
= & \frac{2 x_{p}}{a_{0}} \int_{-\pi}^{\pi} d \theta \int \frac{\Delta d \Delta}{a_{0}^{2}} f_{\text {pair }}(g, \Delta, \theta, 0) .
\end{aligned}
$$

The integration over $\Delta$ is restricted to the strong pinning region, i.e., $\kappa_{\mathrm{eff}}(\theta)>1$. Rewriting the integration in terms of $\boldsymbol{\Delta}=\left(\Delta_{x}, \Delta_{y}\right)$, we compute the factor

$$
\int_{0}^{\Delta_{x}^{0}} \frac{d \Delta_{x}}{a_{0}} \int_{0}^{\Delta_{y}^{0}} \frac{d \Delta_{y}}{a_{0}}\left[1-\frac{\left(\Delta_{x}\right)^{2}}{\left(\Delta_{x}^{0}\right)^{2}}-\frac{\left(\Delta_{y}\right)^{2}}{\left(\Delta_{y}^{0}\right)^{2}}\right]^{2}=\frac{\pi}{3} \frac{\Delta_{x}^{0} \Delta_{y}^{0}}{a_{0}^{2}}
$$

and obtain the averaged pinning force

$$
\left\langle f_{\text {pair }}(g, \boldsymbol{\Delta})\right\rangle_{\Delta}=\frac{2 x_{p}}{a_{0}^{2}} \frac{6 \pi(\kappa \bar{C})^{2}}{\left[-f_{p}^{\prime \prime \prime}\left(r_{m}\right)\right]} \frac{\left(g-g_{0}\right)^{2}}{(1+g)^{4}} \frac{\Delta_{x}^{0} \Delta_{y}^{0}}{a_{0}^{2}} .
$$

Keeping systematically the corrections in the jump position $r_{m}+O\left(\boldsymbol{\Delta}^{2}\right)$ is equivalent to replacing $b=x_{p} \sin \phi+O\left(\boldsymbol{\Delta}^{2}\right)$ in the integration leading to the Eq. (110). Carrying out the integration of the additional $O\left(\boldsymbol{\Delta}^{2}\right)$ term would contribute with a quartic correction $\propto \Delta^{4}$ to Eq. (111) which we ignore here. With $x_{p} \sim \xi$ and the results for the maximum mismatches $\Delta_{x}^{0}$, $\Delta_{y}^{0}$ in Eqs. (98) and (103), we obtain a parametric estimate for the pinning force originating from defect pairs in the form

$$
\left\langle f_{\text {pair }}(g, \boldsymbol{\Delta})\right\rangle_{\boldsymbol{\Delta}} \sim \frac{\xi^{4}}{a_{0}^{4}}\left(g-g_{0}\right)^{3}\left(g+g_{0}\right)^{1 / 2} f_{p},
$$

with the following interpretation: the defect pair induces the pinning force $\sim f_{p}$ rescaled by the factor $\left(g-g_{0}\right)^{2}$ that accounts for the distance between defects, a factor $\xi^{2} / a_{0}^{2} \sim$ $S_{\text {trap }} / a_{0}^{2}$ that represents the areal fraction where vortices are trapped, and the additional factor $\xi^{2} / a_{0}^{2}$ together with the distance-dependent factor $\left(g-g_{0}\right)\left(g+g_{0}\right)^{1 / 2}$ that derive from the constraint on the mismatch $\boldsymbol{\Delta}$. The result (112) is the basis for the evaluation of the pinning force due to defect pairs at different separations in Sec. III C.

\section{SUMMARY AND CONCLUSION}

We have extended the strong pinning paradigm into the weak pinning domain by accounting for correlations between defects. The most relevant correlations arise from defect pairs - they reduce the critical Labusch parameter (or pinning strength) $\kappa \sim-e_{p}^{\prime \prime} / \bar{C}$ for strong pinning from its standard value $\kappa_{c}=1$ to $\kappa_{c \text {,pairs }}=1 / 2$. When decreasing the individual defect's pinning strength $\kappa$ towards the critical value $\kappa_{c}$, the strong pinning force density vanishes as $F_{\text {pin }} \propto n_{p}(\kappa-1)^{2}$ and strong pair-pinning takes over. Upon a further decrease of $\kappa$, the pair-induced strong pinning force density $F_{\text {pin }}$ scales with $n_{p}^{2}$ and vanishes at $\kappa=1 / 2$ according to $F_{\text {pin }} \propto n_{p}^{2}(\kappa-$ $1 / 2)^{4}$. The contributions to $F_{\text {pin }}$ from higher-order correlations between $n$ defects scale as $\left(n_{p}\right)^{n}$ and quickly become irrelevant, with the dominant contribution to the pinning force density being taken over by weak-collective pinning with $F_{\text {pin }} \propto n_{p}^{2} \kappa^{3}$ as $\kappa$ drops below $1 / 2$.

The origin of the pair-induced strong pinning condition $\kappa>1 / 2$ is easily understood-for two defects that overlap in position, their joint pinning strength doubles and they reach the strong pinning criterion $2 \kappa>1$. The substantial enhancement in pinning strength remains in place for small defect pairs that are separated at most by $\sim \xi$ and $\sim a_{0}$ in transverse and longitudinal (field) directions and act on the same vortex. However, this is not the full story: with $\kappa>1 / 2$ approaching unity from below, pairs separated by transverse distances beyond $a_{0}$ can constitute a strong pinning pair as well. These pairs, rather than pinning the same vortex, will pin different vortices. The interaction between these two defect vortex entities is transmitted by the elastic properties of the vortex lattice, specifically, the static nonlocal Green's function $G_{\alpha \beta}(\mathbf{R}, z)$, and determines the effective pinning strength of the extended pair which is smaller than the one of a small pair.

The Green's function $G_{\alpha \beta}(\mathbf{R}, z)$ describes the displacement field $\mathbf{u}(\mathbf{R}, z)$ for a $\delta$ force acting at the origin and hence the distortion at the site of a second defect that is positioned a distance $(\mathbf{R}, z)$ away from the first defect. In our analysis, we have simplified the expression for the Green's function and considered its diagonal, transverse part $G(\mathbf{R}, z)$; the result, shown in Fig. 3, exhibits a sharp asymmetric and structured peak in the shape of a dumbbell. This complex real-space structure has not been considered before and is expected to be present in the full expression for the response matrix $G_{\alpha \beta}(\mathbf{R}, z)$ as well.

For our extended pairs, the effective Labusch parameter or pinning strength $\kappa_{\text {eff }}$, rather than simply doubling $\kappa$, scales as $\kappa_{\text {eff }} \sim(1+g) \kappa$, with $g=g(\mathbf{R}, z)=G(\mathbf{R}, z) / G(0,0)<1$; hence, the partner defect contributes to the strong pinning with a reduced weight. Extended pairs within a distance determined by the condition $g(\mathbf{R}, z)>1 / \kappa-1 \equiv g_{0}(\kappa)$ thus potentially contribute to strong pair-pinning; geometric considerations refine this analysis and produce an effective Labusch parameter $\kappa_{\text {eff }}(g, \boldsymbol{\Delta})$ that depends on distance (through $g$ ) and on the misfit $\boldsymbol{\Delta}$ between the defect pair and the vortex lattice, with a finite $\Delta$ further reducing the effective pinning strength $\kappa_{\text {eff }}$; see Eq. (104).

The effective Labusch parameter $\kappa_{\text {eff }}(g, \boldsymbol{\Delta})$ exhibits a nontrivial angular dependence encoded in the direction of $\boldsymbol{\Delta}$. While for isotropic single-defect pinning, strong pinning jumps appear near the inflection points arranged in a circle of radius $r_{m}$, for an anisotropic potential as in Eq. (83), strong pinning jumps appear on arcs that grow with decreasing elasticity $\bar{C}$ or increasing pinning strength $e_{p}$ as illustrated in Fig. 10. The direction away from the defect center where these arcs make their first appearance depends, besides the direction of $\Delta$, on the detailed shape of the pinning potential; see Eq. (105).

With contributions to strong pair-pinning arising both from small pairs pinning one vortex and extended pairs pinning two separated (and relatively misfitted) vortices, the question arises about their relative total weight. It turns out, that the extended-pair force decreases with the scaled distance $\tilde{\rho}=$ $\left[R^{2}+\left(a_{0}^{2} / 16 \pi \lambda^{2}\right) z^{2}\right]^{1 / 2}$ as $\propto \tilde{\rho}^{-7 / 2}$, that makes the smallpair contribution (originating from pairs in a small volume $\xi^{2} a_{0}$ ) dominate the strong pair-pinning force density $F_{\text {clust }}$ in Eq. (32).

As follows from the above discussion, the elastic properties of the vortex lattice take an important role in the calculation 
of the strong pair-pinning force. Furthermore, they also define the dominance of strong pair-pinning over weak-collective pinning that is reduced by the factor $\left(a_{0} / \lambda\right)^{2}$. This reduction is a consequence of the nonlocal interaction between vortices producing a dispersion in $c_{44}(k)$. While collective pinning in the nondispersive regime (with a Larkin length $R_{c}>\lambda$ ) involves a "stiff" lattice with $c_{44}(k=0)=B^{2} / 4 \pi$, the small defect pairs involve the soft lattice with $c_{44}(k)=B^{2} / 4 \pi \lambda^{2} k^{2}$; with the relevant $k \sim K_{\mathrm{BZ}} \approx \sqrt{4 \pi} / a_{0}$, the lattice is softer by a factor $\sim a_{0}^{2} / \lambda^{2}$ and hence pair pinning is stronger. The large factor $\lambda^{2} / a_{0}^{2}$ also guarantees, that strong pair-pinning is larger than weak-collective pinning deep in the dispersive regime where the lattice becomes softer.

It is interesting to compare the situation described above with the one studied by Fisher [32]: Focusing on weak defects and large dimensions $D>4$, it turns out that weak-collective pinning is ineffective due to the fast spatial relaxation of the manifold's distortions. Pinning then is exclusively due to rare configurations appearing in the random pinning landscape. The possible existence of a pinning threshold in higher dimensions and for different types of short- and long-range elasticities was recently discussed in Ref. [39]. In our analysis, we start from the opposite limit, strong defects that pin the elastic manifold (here, vortices) individually. Upon decreasing the pinning strength $\kappa$ below unity, we loose the strong pinning of individual defects and would expect weak-collective pinning to take over in $D=3$, where distortions decay slowly, proportional to the inverse distance. Instead, due to the nonlocal interaction between vortices producing a dispersive elastic response, we find that specific rare events, small defect pairs, take over and produce the leading pinning mechanism. In a nondispersive elastic medium, the stiffening at large scales is absent and the two types of pinning, rare and collective, come with equal (parametric) weight.

While weak-collective pinning arises from typical fluctuations in the defect distribution, strong pair or cluster pinning arises from rare fluctuations. In reality, both types of fluctuations coexist and hence simultaneously contribute to the pinning force density $F_{\text {pin }}$. Similar to the addition of resistivities arising from different scattering mechanisms in the Matthiessen rule describing metallic transport, the pinningforce densities from different pinning mechanisms should be added up to the total pinning force $F_{\text {pin }} \approx F_{\text {coll }}+F_{\text {clust }}$ when describing the transport in a superconductor. However, given the inductive response of a superconductor, this corresponds to an addition of (critical) currents rather than voltages. Microscopically, comparing the distance between small pairs, $d_{\text {pairs }} \sim\left[n_{p}\left(n_{p} a_{0} \xi^{2}\right)\right]^{-1 / 3}$, with the size of the collective pinning volume $V_{c} \sim L_{c} R_{c}^{2} \sim\left(\lambda / a_{0}\right) R_{c}^{3}$, one notes that $V_{c}$ contains many pairs. Hence, when dragging a vortex system slowly over the pinning landscape, one should observe a complex stick-slip type motion where small slips of individual vortices depinning from defect pairs combine with large slips of collectively pinned vortex bundles. It would be interesting to observe the motion of such a pinned vortex system in a numerical simulation. Another future topic of interest is the further investigation of the real-space structure of the Green's function $G_{\alpha \beta}(\mathbf{R}, z)$, both theoretically as well as experimentally. In particular, it would be interesting to come up with a proposal for an experiment that is sensitive to the nontrivial dumbbell structure of the peak in the response function.

\section{ACKNOWLEDGMENTS}

We thank Alexei Koshelev and Roland Willa for inspiring discussions. M.B. acknowledges financial support from the Swiss National Science Foundation, Division II.

\section{APPENDIX A: GREEN'S FUNCTION IN THE DISPERSIVE REGIME}

We discuss the derivation of the interpolation formula (8) for the diagonal Green's function in the dispersive region (short distances). We consider the simplified model of the vortex lattice elasticity where we drop the longitudianal part in Eq. (3) containing the large compression modulus $c_{11}(\mathbf{k})>$ $c_{66}$; furthermore, we replace the transverse projector by unity and study the diagonal Green's function $G_{\alpha \beta}(\rho)=G(\rho) \delta_{\alpha \beta}$. The integration in reciprocal space then reads

$$
G(\mathbf{R}, z)=\int_{K<K_{\mathrm{BZ}}} \frac{d^{2} \mathbf{K} d k_{z}}{(2 \pi)^{3}} \frac{e^{i \mathbf{K} \cdot \mathbf{R}} e^{i k_{z} z}}{c_{66} K^{2}+c_{44}(\mathbf{k}) k_{z}^{2}}
$$

with the nondispersive shear modulus $c_{66}$, the dispersive tilt modulus $c_{44}(\mathbf{k}) \approx c_{44}(k=0) /\left(1+\lambda^{2} k^{2}\right)$, and $c_{66} / c_{44}=$ $a_{0}^{2} / 16 \pi \lambda^{2}$ (we write $\left.c_{44}(k=0) \equiv c_{44}\right)$. We first perform the complex integration over $k_{z}$ (extended to infinity) with a pole at

$$
k_{z}=i K \frac{\left(1+\lambda^{2} K^{2}\right)^{1 / 2}}{\left(\lambda^{2} K^{2}+c_{44} / c_{66}\right)^{1 / 2}} .
$$

We drop the term $\lambda^{2} K^{2}$ in the denominator that provides a numerical correction to the large ratio $c_{44} / c_{66}$ when $K<K_{\mathrm{BZ}} \approx$ $\sqrt{4 \pi} / a_{0}$ is residing within the Brillouin zone. The Green's function then takes the form

$$
\begin{aligned}
G(\mathbf{R}, z) \approx & \frac{\lambda}{2 \sqrt{c_{44} c_{66}}} \int \frac{d^{2} \mathbf{K}}{(2 \pi)^{2}} e^{i \mathbf{K} \cdot \mathbf{R}} \frac{\left(1+\lambda^{2} K^{2}\right)^{1 / 2}}{\lambda K} \\
& \times \exp \left[-\frac{a_{0} z}{4 \sqrt{\pi} \lambda} K\left(1+\lambda^{2} K^{2}\right)^{1 / 2}\right] .
\end{aligned}
$$

We assume a small distance $\rho=(\mathbf{R}, z)$ within the ellipse $R^{2}+$ $\left(a_{0}^{2} / 16 \pi \lambda^{2}\right) z^{2} \ll \lambda^{2}$ [see Eq. (7) for the opposite limit]. We first focus on the contribution from $\lambda K \gg 1$ and approximate $\left(1+\lambda^{2} K^{2}\right)^{1 / 2} \rightarrow \lambda K$ in Eq. (A3), that provides us with the dispersive approximation

$$
\begin{aligned}
G_{\mathrm{d}}(\mathbf{R}, z)= & \frac{\lambda}{2 \sqrt{c_{44} c_{66}}} \int \frac{d^{2} \mathbf{K}}{(2 \pi)^{2}} e^{i \mathbf{K} \cdot \mathbf{R}} \exp \left[-\frac{a_{0} z K^{2}}{4 \sqrt{\pi}}\right] \\
& =\frac{1 / \sqrt{4 \pi}}{a_{0} \sqrt{c_{44} c_{66}}} \frac{\lambda}{z} \exp \left[-\frac{\sqrt{\pi} R^{2}}{a_{0} z}\right] .
\end{aligned}
$$

Next, we account for the difference between the full expression (A3) and the dispersive approximation (A4); we split this difference into two terms $\delta G_{<}$and $\delta G_{>}$arising from small 
$\left(K<K_{0}\right)$ and large $\left(K>K_{0}\right)$ momenta,

$$
\begin{aligned}
\delta G_{<}= & \frac{\lambda}{2 \sqrt{c_{44} c_{66}}} \int_{K<K_{0}} \frac{d^{2} \mathbf{K}}{(2 \pi)^{2}} \frac{e^{i \mathbf{K} \cdot \mathbf{R}}}{\lambda K} \\
& \times\left\{\left(1+\lambda^{2} K^{2}\right)^{1 / 2} \exp \left[-\frac{a_{0} z}{4 \sqrt{\pi} \lambda} K\left(1+\lambda^{2} K^{2}\right)^{1 / 2}\right]\right. \\
& \left.-\lambda K \exp \left[-\frac{a_{0} z K^{2}}{4 \sqrt{\pi}}\right]\right\},
\end{aligned}
$$

and a corresponding expression for $\delta G_{>}$covering the remaining region $K_{0}<K<K_{\mathrm{BZ}}$. The scale $K_{0}$ is chosen such as to satisfy $\lambda^{-1} \ll K_{0} \ll R^{-1}$ as well as $\lambda^{-1} \ll K_{0} \ll\left(a_{0} z\right)^{-1 / 2}$, consistent with the assumption that $R \ll \lambda$ and $z \ll \lambda^{2} / a_{0}$.

In carrying out the integration over small momenta $K<$ $K_{0}$, we note that the arguments in the exponentials of Eq. (A6) remain small since $K R<K_{0} R \ll 1$ and $K_{0} \ll\left(a_{0} z\right)^{-1 / 2}$; performing the integration over angles then provides us with

$$
\begin{aligned}
\delta G_{<} & =\frac{1 / 4 \pi}{\sqrt{c_{44} c_{66}}} \int_{0}^{K_{0}} d K\left[\left(1+\lambda^{2} K^{2}\right)^{1 / 2}-\lambda K\right] \\
& \approx \frac{1 / 16 \pi}{\lambda \sqrt{c_{44} c_{66}}}\left[1+2 \ln \left(2 K_{0} \lambda\right)\right],
\end{aligned}
$$

where we have used that $\lambda K_{0} \gg 1$ in the last relation.

For large momenta $K_{0}<K<K_{\mathrm{BZ}}$, we rewrite the integral in the form

$$
\begin{aligned}
\delta G_{>}= & \frac{\lambda}{2 \sqrt{c_{44} c_{66}}} \int_{K_{0}<K<K_{\mathrm{BZ}}} \frac{d^{2} \mathbf{K}}{(2 \pi)^{2}} \frac{e^{i \mathbf{K} \cdot \mathbf{R}}}{\lambda K} e^{-a_{0} z K^{2} / 4 \sqrt{\pi}} \\
& \times\left\{\sqrt{1+\lambda^{2} K^{2}} e^{-\frac{a_{0} z}{4 \sqrt{\pi} \lambda^{2}}\left[\lambda K\left(\sqrt{1+\lambda^{2} K^{2}}-\lambda K\right)\right]}-\lambda K\right\} .
\end{aligned}
$$

Since $K \lambda>K_{0} \lambda \gg 1$, we can expand the square roots; furthermore the $K$ integration is cut off by the exponentials, either via $K \sim\left(a_{0} z\right)^{-1 / 2}<K_{\mathrm{BZ}}$ or by $K \sim R^{-1}<K_{\mathrm{BZ}}$ since $R, z>a_{0}$, hence the $\mathrm{BZ}$ cutoff at $K_{\mathrm{BZ}}$ can be replaced by infinity,

$$
\delta G_{>} \approx \frac{1}{4 \lambda \sqrt{c_{44} c_{66}}} \int_{K>K_{0}} \frac{d^{2} \mathbf{K}}{(2 \pi)^{2}} \frac{e^{i \mathbf{K} \cdot \mathbf{R}}}{K^{2}} e^{-a_{0} z K^{2} / 4 \sqrt{\pi}} .
$$

In the evaluation along the longitudinal direction, we expand the integrand in the small parameter $\left(a_{0} z\right)^{1 / 2} K_{0} \ll 1$ and obtain the result

$$
\delta G_{>}(\mathbf{R}=0, z) \approx \frac{1 / 8 \pi}{\lambda \sqrt{c_{44} c_{66}}}\left[-\gamma / 2+\ln \frac{2 \pi^{1 / 4}}{K_{0 \sqrt{a_{0} z}}}\right]
$$

with the Euler-Mascheroni constant $\gamma$. Along the transverse direction, we expand in $K_{0} R \ll 1$ and find that

$$
\delta G_{>}(\mathbf{R}, z=0) \approx \frac{1 / 8 \pi}{\lambda \sqrt{c_{44} c_{66}}}\left[-\gamma+\ln \frac{2}{K_{0} R}\right] .
$$

We combine these results to arrive at the interpolation formula for a general distance $\rho=(\mathbf{R}, z)$ within the ellipse

$$
\delta G_{>}(\mathbf{R}, z) \approx \frac{1 / 16 \pi}{\lambda \sqrt{c_{44} c_{66}}}\left[-2 \gamma+\ln \frac{4 / K_{0}^{2}}{R^{2}+a_{0} z / e^{\gamma} \sqrt{\pi}}\right] .
$$

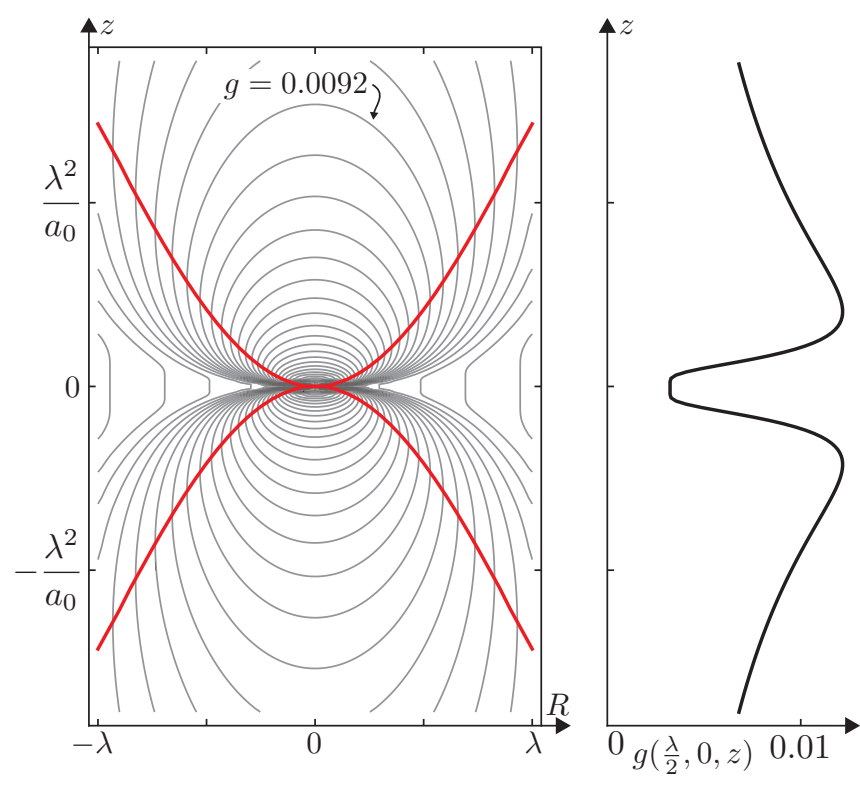

FIG. 11. Left: contour plot of the rescaled Green's function $g(x=R, 0, z)=G(R, 0, z) / G(\mathbf{0})$ evaluated for $\lambda=10 a_{0}$ illustrating the dumbbell structure of the central peak; axes are not to scale. The ridge marking the maximum of $g$ when increasing $z$ at fixed $R<\lambda$ has a parabolic shape (red lines). Subsequent contours are separated by a factor $2^{1 / 4}$. Right: Interpreting the Green's function $G$ as providing the displacement field due to a point force in the origin, the ratio $g(x=\lambda / 2,0, z)$ follows the profile of a vortex placed at a distance $\lambda / 2$ away from the force center. The ridges in $g$ manifest as maxima in the vortex displacement away from $z=0$.

Finally, summing up the contributions $G_{\mathrm{d}}+\delta G_{<}+\delta G_{>}$provides us with the result in Eq. (8) (note that the momentum $K_{0}$ drops out from the final expression); it provides us with the peak in $G(\mathbf{R}, z)$ at small distances with its dumbbell structure that is illustrated in Fig. 11.

\section{APPENDIX B: NUMERICAL EVALUATION OF THE PINNING FORCE}

Our numerical evaluation of the pinning force $f_{\text {pair }}$ $(g, \Delta, \theta, b)$ (see Sec. III F) makes use of the numerical solution for the vortex displacements $\mathbf{u}_{1}, \mathbf{u}_{2}$ in the two-defect problem Eq. (39). We first reformulate the latter in terms of a minimization problem for the total energy $e_{\text {pin }}^{\text {pair }}$ of the two defect system described by the mean asymptotic vortex position $\mathbf{x}$, the fixed mismatch $\Delta$ and vortex-tip displacements $\mathbf{u}_{1}, \mathbf{u}_{2}$,

$$
\begin{aligned}
e_{\text {pin }}^{\text {pair }}\left(\mathbf{x}, \Delta ; \mathbf{u}_{1}, \mathbf{u}_{2}\right) \equiv & e_{p}\left(\mathbf{x}+\Delta / 2+\mathbf{u}_{1}\right)+e_{p}\left(\mathbf{x}-\Delta / 2+\mathbf{u}_{2}\right) \\
& +\frac{\bar{C}}{2\left(1-g^{2}\right)}\left[\mathbf{u}_{1}^{2}+\mathbf{u}_{2}^{2}-2 g \mathbf{u}_{1} \cdot \mathbf{u}_{2}\right],
\end{aligned}
$$

such that setting $\partial e_{\text {pin }}^{\text {pair }} / \partial \mathbf{u}_{1,2}=0$ reproduces Eq. (39). By rewriting $\mathbf{u}_{1}^{2}+\mathbf{u}_{2}^{2}-2 g \mathbf{u}_{1} \cdot \mathbf{u}_{2}=\left(\mathbf{u}_{1}-\mathbf{u}_{2}\right)^{2}+2(1-g)$ $\mathbf{u}_{1} \cdot \mathbf{u}_{2}$, we note that in the limit $g=1$ (defects pinning the same vortex at the same height $z$, such that $\mathbf{u}_{1}=\mathbf{u}_{2}$ ), the elastic term in Eq. (B1) remains regular and reduces to $\bar{C} \mathbf{u}_{1}^{2} / 2$; Eq. (B1) then describes the interaction of a single vortex with 


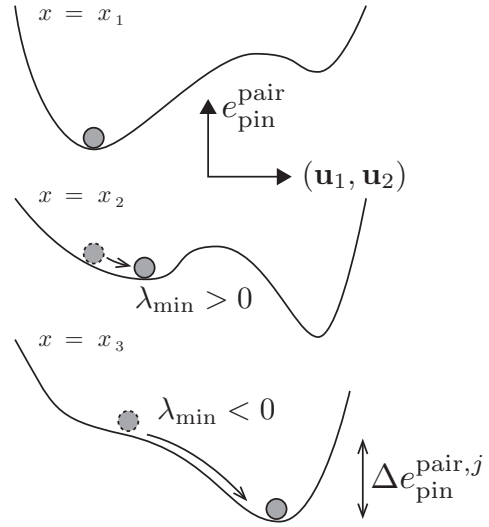

FIG. 12. Minimization of the pinning energy (B1). Given the position $\mathbf{x}=(x, b)$ of the vortex system with respect to the defects, the pinning energy $e_{\mathrm{pin}}^{\text {pair }}$ is a function of the four-dimensional vector $\left(\mathbf{u}_{1}, \mathbf{u}_{2}\right)$. The pictures sketch the qualitative changes in the shape of $e_{\text {pin }}^{\text {pair }}$ when increasing the mean vortex position through $x_{1}<x_{2}<x_{3}$. The neighborhood of each local minimum is characterized by positive eigenvalues $\lambda$ of the matrix of second derivatives of $e_{\mathrm{pin}}^{\mathrm{pair}}$. The smallest eigenvalue going negative indicates the disappearance of a local minimum and triggers a jump in the location of the occupied minimum.

a defect potential given by the superposition of two pinning potentials shifted by the mismatch vector $\Delta$ from each other.

We evaluate the critical force for a quasistatic vortex lattice pushed to the right: For every asymptotic vortex trajectory $\mathbf{x}=$ $(x, b)$ with fixed impact parameter $b$, we increase the position $x$ in small steps, and minimize the pinning energy at each step using the solution from the previous position as the starting point. Starting far to the left from both defects, the contribution of the pinning energies $e_{p}\left(\mathbf{x} \pm \Delta / 2+\mathbf{u}_{1,2}\right)$ is negligible and the unique solution describes a system of unperturbed vortices with $\mathbf{u}_{1,2} \approx \mathbf{0}$. On approaching the defects, the vortices deform and multiple minima develop (see Fig. 12); a small stepwise increase of $x$ ensures that the occupied minimum develops continuously (see below for details on the minimization algorithm). At the point where the minimum disappears, the solution changes abruptly, with the associated jump in the pinning energy contributing to the average pinning force.

Depending on the specific setting of the problem, the system undergoes a number of jumps in energy with increasing $x$ (see Fig. 10), where the fictitious vortex undergoes zero, one, or two jumps depending on the angle $\theta$ and impact parameter $b$. Going beyond the transition to strong pinning with $\kappa>1$, the two-vortex system may undergo up to four jumps associated with the pinning and depinning events of two vortices hitting the two strong pinning defects. The numerical procedure then provides a convenient way to evaluate the average pinning force for the case with an arbitrary number of jumps in energy (even though only the cases with zero, one, or two jumps have been discussed in the analytic part of this paper).

As for the single-defect case, it turns out that the pinning force exerted on the vortices can be expressed as the gradient of the total pinning energy. Indeed, taking the gradient of $e_{\text {pin }}^{\text {pair }}(\mathbf{x}) \equiv e_{\text {pin }}^{\text {pair }}\left[\mathbf{x}, \boldsymbol{\Delta} ; \mathbf{u}_{1}(\mathbf{x}), \mathbf{u}_{2}(\mathbf{x})\right]$ defined through Eq. (B1), we find

$$
\nabla_{\mathbf{x}} e_{\mathrm{pin}}^{\mathrm{pair}}(\mathbf{x})=\frac{\partial e_{\mathrm{pin}}^{\mathrm{pair}}}{\partial \mathbf{x}}+\sum_{i=1,2} \frac{\partial e_{\mathrm{pin}}^{\mathrm{pair}}}{\partial \mathbf{u}_{i}} \frac{\partial \mathbf{u}_{i}}{\partial \mathbf{x}}=\frac{\partial e_{\mathrm{pin}}^{\mathrm{pair}}}{\partial \mathbf{x}},
$$

where the partial derivative $\partial e_{\mathrm{pin}}^{\mathrm{pair}} / \partial \mathbf{x}$ is taken at fixed $\mathbf{u}_{1}, \mathbf{u}_{2}$. The term in Eq. (B2) involving the $\mathbf{u}$ derivatives vanishes since $\partial e_{\text {pin }}^{\text {pair }} / \partial \mathbf{u}_{1,2}=0$ at the minimum. Taking the partial $\mathbf{x}$ derivative in Eq. (B1) provides us with $-\nabla_{\mathbf{x}} e_{\text {pin }}^{\text {pair }}=\mathbf{f}_{p}[\mathbf{x}+$ $\left.\Delta / 2+\mathbf{u}_{1}(\mathbf{x})\right]+\mathbf{f}_{p}\left[\mathbf{x}-\boldsymbol{\Delta} / 2+\mathbf{u}_{2}(\mathbf{x})\right]$, which is precisely the pinning force exerted by the two defects on the distorted vortices. The $x$-averaged pinning force along the trajectory $\mathbf{x}=(x, b)$ is then written as

$$
f_{\text {pair }}(g, \Delta, b)=\int \frac{d x}{a_{0}} \mathbf{e}_{x} \cdot\left[-\nabla_{\mathbf{x}} e_{\text {pin }}^{\text {pair }}\right]=-\sum_{j} \frac{\Delta e_{\text {pin }}^{\text {pair }}, j}{a_{0}},
$$

with $\quad \Delta e_{\text {pin }}^{\text {pair }, j}=\lim _{\varepsilon \rightarrow 0}\left[e_{\text {pin }}^{\text {pair }}\left(x_{j}-\varepsilon, b\right)-e_{\text {pin }}^{\text {pair }}\left(x_{j}+\varepsilon, b\right)\right]$ quantifying the energy jump at the position $x_{j}$. Integrating $\partial e_{\mathrm{pin}}^{\mathrm{pair}} / \partial y$ would in general give a nonvanishing contribution to the pinning force in the $y$ direction; it is, however, compensated by the configuration with $\Delta \rightarrow-\boldsymbol{\Delta}$ and $b \rightarrow-b$ after averaging.

The result of this numerical evaluation is compared with the analytic result (108) in Fig. 8 for the case of marginally strong pair pinning $g-g_{0} \ll 1$ and shows a very good agreement with the analytic result even at large values of the mismatch $\Delta$ of order $\xi$ at angles $\theta$ close to $\pi / 2$, in which case the theoretical estimates made in Sec. III E do not guarantee the validity of the perturbative approach. For a Lorentzian shape potential and parameters used in Fig. 8 with $\theta=\pi / 2$, we find that the scaling factor in Eq. (102) assumes a value $\left[\left(g-g_{0}\right) /(1+g)\left(g+g_{0}\right)\right]^{1 / 2} \approx 0.17$ and the prefactor contributes a factor $\approx 4.46$, such that $\Delta_{y}^{0} \approx 0.78 \xi$.

\section{Numerical minimization}

The main challenge in the minimization of the two-defect pinning energy in Eq. (B1) is to properly track the local minimum representing the current state of the vortex (the occupied branch) and to ensure that the minimization algorithm does not overshoot to another minimum.

We define $\mathbf{u}=\left(u_{1, x}, u_{1, y}, u_{2, x}, u_{2, y}\right)$ and minimize the function $e_{\mathrm{pin}}^{\text {pair }}(\mathbf{u}, \mathbf{x})$ [see Eq. (B1)] with respect to $\mathbf{u}$. For a fixed asymptotic position $\mathbf{x}$, we use Newton's method to iterate $\mathbf{u}$,

$$
\mathbf{u}_{i+1}=\mathbf{u}_{i}-\gamma H\left(\mathbf{u}_{i}\right)^{-1} \nabla_{\mathbf{u}} e_{\text {pin }}^{\text {pair }}(\mathbf{u}, \mathbf{x}),
$$

as long as the matrix of second derivatives $H_{\alpha \beta}=$ $\partial^{2} e_{\text {pin }}^{\text {pair }} / \partial u_{\alpha} \partial u_{\beta}$ evaluated at $\mathbf{u}_{i}$ remains positive-definite. The parameter $\gamma$ is chosen to bound the step size $\left|\mathbf{u}_{i+1}-\mathbf{u}_{i}\right|<\delta u_{\max }$ to a predefined maximum value $\delta u_{\max }$. The method converges if the initial guess $\mathbf{u}_{0}$ lies close to a local minimum and the new minimum is used as an initial guess for the minimization after changing the parameter $\mathbf{x}$.

The appearance of at least one negative eigenvalue of $H(\mathbf{u})$ during the minimization signals the disappearance of the local minimum and triggers the jump to another 
minimum. Minimization through this region is performed using the Nelder-Mead algorithm with the initial simplex size set to $\delta u_{\max }$. Once the positive-definite region in the neighborhood of the new minimum is reached, the minimization procedure switches back to Newton's method.

Obtaining the size of the energy jumps to the desired accuracy requires precise determination of the jump points $\mathbf{x}_{j}=\left(x_{j}, b\right)$ where the currently occupied local minimum disappears. This is achieved by repeated interval halving: assume that for $\mathbf{x}=\left(x_{0}, b\right)$ the Newton minimization converged to a local minimum $\mathbf{u}_{0}$, that is used as a starting point for the minimization at the next position $\mathbf{x}=\left(x_{0}+\delta x, b\right)$. The appearance of a region with a negative eigenvalue of the Hessian during this minimization indicates the presence of the jump point $x_{j}$ in the interval $\left(x_{0}, x_{0}+\delta x\right)$. Another minimization is thus performed for $x=x_{0}+\delta x / 2$ that reduces the interval either to $\left(x_{0}, x_{0}+\delta x / 2\right)$ (if the negative eigenvalue region appears during the minimization) or $\left(x_{0}+\delta x / 2, x_{0}+\delta x\right)$. The further iteration of this procedure locates the jump point to the required precision.
[1] A. A. Abrikosov, On the magnetic properties of superconductors of the second group, Zh. Eksp. Teor. Fiz. 32, 1442 (1957) [JETP 5, 1174 (1957)].

[2] F. Bloch, Zur Theorie des Austauschproblems und der Remanenzerscheinung der Ferromagnetika, Z. Physik 74, 295 (1932).

[3] L. D. Landau and E. Lifshitz, On the theory of the dispersion of magnetic permeability in ferromagnetic bodies, Phys. Z. Sowjet 8, 153 (1935).

[4] R. Labusch, Calculation of the critical field gradient in type-II superconductors, Cryst. Lattice Defects 1, 1 (1969).

[5] A. I. Larkin and Y. N. Ovchinnikov, Pinning in type II superconductors, J. Low Temp. Phys. 34, 409 (1979).

[6] C. Kittel, Physical theory of ferromagnetic domains, Rev. Mod. Phys. 21, 541 (1949).

[7] G. Blatter, M. V. Feigel'man, V. B. Geshkenbein, A. I. Larkin, and V. M. Vinokur, Vortices in high-temperature superconductors, Rev. Mod. Phys. 66, 1125 (1994).

[8] T. Nattermann and S. Scheidl, Vortex-glass phases in type-II superconductors, Adv. Phys. 49, 607 (2000).

[9] A. I. Larkin and Yu. N. Ovchinnikov, Electrodynamics of inhomogeneous type-II superconductors, Zh. Eksp. Teor. Fiz. 65, 1704 (1974) [JETP 38, 854 (1974)].

[10] A. Schmid and W. Hauger, On the theory of vortex motion in an inhomogeneous superconducting film, J. Low Temp. Phys. 11, 667 (1973).

[11] A. I Larkin, Effect of inhomogeneities on the structure of the mixed state of superconductors, Zh. Eksp. Teor. Fiz. 58, 1466 (1970) [JETP 31, 784 (1970)].

[12] T. Giamarchi and P. Le Doussal, Elastic Theory of Pinned Flux Lattices, Phys. Rev. Lett. 72, 1530 (1994).

[13] T. Giamarchi and P. Le Doussal, Elastic theory of flux lattices in the presence of weak disorder, Phys. Rev. B 52, 1242 (1995).

[14] S. E. Korshunov, Replica symmetry breaking in vortex glasses, Phys. Rev. B 48, 3969 (1993).

[15] W. Kleemann, Universal domain wall dynamics in disordered ferroic materials, Annu. Rev. Mater. Research 37, 415 (2007).

[16] J. Gorchon, S. Bustingorry, J. Ferré, V. Jeudy, A. B. Kolton, and T. Giamarchi, Pinning-Dependent Field-Driven Domain Wall Dynamics and Thermal Scaling in an Ultrathin $\mathrm{Pt} / \mathrm{Co} / \mathrm{Pt}$ Magnetic Film, Phys. Rev. Lett. 113, 027205 (2014).

[17] V. Jeudy, A. Mougin, S. Bustingorry, W. Savero Torres, J. Gorchon, A. B. Kolton, A. Lemaître, and J.-P. Jamet, Universal Pinning Energy Barrier for Driven Domain Walls in Thin Ferromagnetic Films, Phys. Rev. Lett. 117, 057201 (2016).

[18] P. A. Lee and T. M. Rice, Electric field depinning of charge density waves, Phys. Rev. B 19, 3970 (1979).
[19] S. Brazovskii and T. Nattermann, Pinning and sliding of driven elastic systems: From domain walls to charge density waves, Adv. Phys. 53, 177 (2004).

[20] P. Chauve, T. Giamarchi, and P. Le Doussal, Creep and depinning in disordered media, Phys. Rev. B 62, 6241 (2000).

[21] S. Brazovskii, Intrinsic defects and plasticity in charge and spin density waves, in Physics and Chemistry of Low-Dimensional Inorganic Conductors, edited by C. Schlenker, J. Dumas, M. Greenblatt, and S. van Smaalen, Vol. 354 (Springer, New York, 1996), p. 465.

[22] S. Brazovskii and A. Larkin, Nonlinear conduction of sliding electronic crystals: Charge and spin density waves, J. Phys. IV France 9, Pr10-77 (1999).

[23] A. U. Thomann, V. B. Geshkenbein, and G. Blatter, Vortex dynamics in type-II superconductors under strong pinning conditions, Phys. Rev. B 96, 144516 (2017).

[24] R. Willa, M. Marziali Bermúdez, and G. Pasquini, Thermal hysteresis of the Campbell response as a probe for bulk pinning landscape spectroscopy, Phys. Rev. B 98, 184520 (2018).

[25] R. Willa, A. E. Koshelev, I. A. Sadovskyy, and A. Glatz, Peak effect due to competing vortex ground states in superconductors with large inclusions, Phys. Rev. B 98, 054517 (2018).

[26] M. Buchacek, R. Willa, V. B. Geshkenbein, and G. Blatter, Persistence of pinning and creep beyond critical drive within the strong pinning paradigm, Phys. Rev. B 98, 094510 (2018).

[27] M. Buchacek, R. Willa, V. B. Geshkenbein, and G. Blatter, Strong pinning theory of thermal vortex creep in type-II superconductors, Phys. Rev. B 100, 014501 (2019).

[28] G. Blatter, V. B. Geshkenbein, and J. A. G. Koopmann, Weak to Strong Pinning Crossover, Phys. Rev. Lett. 92, 067009 (2004).

[29] J. A. G. Koopmann, V. B. Geshkenbein, and G. Blatter, Peak effect at the weak to strong pinning crossover, Physica C 404, 209 (2004), proceedings of the Third European Conference on Vortex Matter in Superconductors at Extreme Scales and Conditions.

[30] G. Blatter and V. B. Geshkenbein, Vortex matter, in Superconductivity: Conventional and Unconventional Superconductors, edited by K. H. Bennemann and J. B. Ketterson (Springer, Berlin, 2008), p. 495.

[31] Y. N. Ovchinnikov and B. I. Ivlev, Pinning in layered inhomogeneous superconductors, Phys. Rev. B 43, 8024 (1991).

[32] D. S. Fisher, Sliding charge-density waves as a dynamic critical phenomenon, Phys. Rev. B 31, 1396 (1985).

[33] R. Willa, V. B. Geshkenbein, and G. Blatter, Probing the pinning landscape in type-II superconductors via Campbell penetration depth, Phys. Rev. B 93, 064515 (2016). 
[34] A. E. Koshelev (private communication).

[35] E. H. Brandt, Elastic energy of the vortex state in type II superconductors. II. Low inductions, J. Low Temp. Phys. 26, 735 (1977).

[36] A. Schmid, A time dependent Ginzburg-Landau equation and its application to the problem of resistivity in the mixed state, Phys. Kondens. Mater. 5, 302 (1966).
[37] J. R. Clem, Simple model for the vortex core in a type-II superconductor, J. Low Temp. Phys. 18, 427 (1975).

[38] A. Tanguya and T. Vettorel, From weak to strong pinning I: A finite size study, Eur. Phys. J. B 38, 71 (2004).

[39] X. Cao, S. Bouzat, A. B. Kolton, and A. Rosso, Localization of soft modes at the depinning transition, Phys. Rev. E 97, 022118 (2018). 